\title{
From the microscopic to the van Hove regime in the $X Y$ chain out of equilibrium
}

\author{
Walter H. Aschbacher* \\ Aix Marseille Université, CNRS, CPT, UMR 7332, 13288 Marseille, France \\ Université de Toulon, CNRS, CPT, UMR 7332, 83957 La Garde, France
}

\begin{abstract}
Using the framework of rigorous algebraic quantum statistical mechanics, we construct the unique nonequilibrium steady state in the isotropic $X Y$ chain in which a sample of arbitrary finite size is coupled by a bond coupling perturbation of arbitrary strength to two infinitely extended thermal reservoirs, and we prove that this state is thermodynamically nontrivial. Moreover, extracting the leading second order contribution to its microscopic entropy production and deriving its entropy production in the van Hove weak coupling regime, we prove that, in the mathematically and physically important $X Y$ chain, the van Hove regime reproduces the leading order contribution to the microscopic regime.
\end{abstract}

Mathematics Subject Classifications (2010) 46L60, 47B15, 82C10, 82C23.

Keywords Open systems; nonequilibrium quantum statistical mechanics; quasifree fermions; Hilbert space scattering theory; nonequilibrium steady state; entropy production; van Hove weak coupling regime.

\section{Introduction}

In recent years, a broad range of important thermodynamic properties of open quantum systems have been successfully derived from first principles within the mathematically rigorous framework of algebraic quantum statistical mechanics. Not only return to equilibrium type phenomena from states close to equilibrium have been explored but also fundamental transport processes in systems far from equilibrium have come within reach. In the latter field, an important role is played by the quasifree fermionic systems since,

*walter.aschbacher@cpt.univ-mrs.fr 


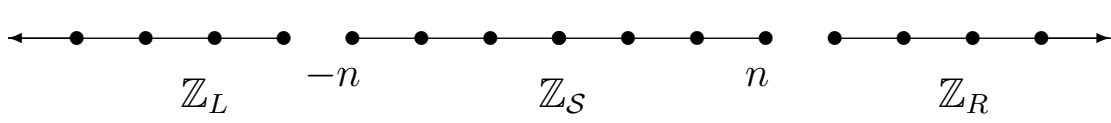

Figure 1: The nonequilibrium setting for the $X Y$ chain.

on one hand, they allow for a powerful description by means of scattering theory on the one-particle Hilbert space on which the fermionic algebra of observables is built being thus ideally suited for a rigorous analysis on many levels. On the other hand, they also constitute a class of systems which are indeed realized in nature. One of the most prominent representatives of this class is the XY spin chain introduced mathematically in 1961 by Lieb et al. [19] who showed that this spin system can be mapped onto a gas of free fermions by using the Jordan-Wigner transformation. Already at the end of the 1960s, the first candidates for a possible physical realization have been identified by Culvahouse et al. [12] and, later, by D'lorio et al. [15] (see also Sologubenko et al. [21] for experiments on more general Heisenberg models). Subsequently, Araki [4] extended the mathematical setup from the finite spin chain to fermions over the two-sided infinite discrete line in the framework of $C^{*}$-dynamical systems and it is this system whose energy transport properties we will study in this paper. In order to do so, we fall back upon the paradigm of the theory of open system by coupling a localized sample to two infinitely extended reservoirs in thermal equilibrium at different temperatures. For this purpose, we cut the two bonds between the sites $\pm n$ and $\pm(n+1)$ of the two-sided discrete line meaning that the coupling strength in the local Hamiltonian between the corresponding sites, initially at value $\lambda=1$, is set to zero. The piece $\mathbb{Z}_{\mathcal{S}}$ between these bonds plays the role of the configuration space of the sample whereas the remaining two half-infinite pieces $\mathbb{Z}_{L}$ and $\mathbb{Z}_{R}$ to its left and right constitute the configuration spaces of the reservoirs, see Figure 1 . Over these configuration spaces, an initial state is prepared as the product of three thermal equilibrium states. The first central object of interest is then the so-called nonequilibrium steady state (NESS) defined by Ruelle [20] as the large time limit of the (averaged) trajectory of the initial state along the fully coupled time evolution. In this setup, the unique NESS has been constructed in Aschbacher and Pillet [6] using Ruelle's scattering approach (for a special case of the XY model, namely for the case of vanishing anisotropy and external magnetic field parameters given in Remark 2 below, this NESS has also been found by Araki and Ho [5] using a different method). It has been proved in [6] that this NESS, henceforth called the XY NESS, is thermodynamically nontrivial in the sense that its entropy production is strictly positive as soon as the system is truly out of equilibrium. In the present paper, we generalize the foregoing situation to couplings of arbitrary strength $\lambda \in \mathbb{R}$. As a first result, we prove that the XY NESS can be embedded into a two-parameter family of NESS parametrized by $\lambda$ and $n$ which, for $\lambda \neq 0$, are all thermodynamically nontrivial. This provides us with a physically richer nonequilibrium situation. In particular, it becomes possible to study the van Hove weak coupling regime $\lambda \rightarrow 0$ of the entropy production of these NESS and compare it to the leading order contribution of the fully microscopic regime. Although one naturally expects that the van Hove regime 
reproduces the leading order contribution to the microscopic regime, this has been proven for few systems only. One of these systems is the so-called simple electronic black box model from Aschbacher et al. [7] (which corresponds to $n=0$ ) and another one is the spin-fermion system from Jakšić et al. [18]. Then, as a second result, and this is the main motivation of the present paper, we prove that this natural expectation is indeed rigorously true in the mathematically and physically important $X Y$ chain out of equilibrium.

The present paper is organized as follows. In Section 2, we introduce the nonequilibrium setting, construct a (family of) unique NESS, and derive an explicit expression for its entropy production. In particular, we prove that, in a true nonequilibrium, this NESS is thermodynamically nontrivial for all nonvanishing couplings and all sample sizes. In Section 3, we extract the leading second order contribution to the microscopic entropy production. In Section 4, we construct the NESS in the van Hove regime and prove that the van Hove entropy production is the leading order contribution to the microscopic entropy production. In Appendix A, some spectral properties of the appearing one-particle Hamiltonians are summarized. In Appendix B, we construct the wave operator needed in the derivation of the microscopic NESS and display some results of the lengthy computations involved in its construction. Finally, in Appendix C, we summarize the van Hove weak coupling theory and derive the necessary decay and positivity properties of the reservoir time correlation function.

\section{Microscopic regime}

We begin this section by summarizing the setting for the system out of equilibrium used in Aschbacher and Pillet [6]. In contradistinction to the presentation there, we skip the formulation of the two-sided $X Y$ chain as a spin system and rather focus directly on the underlying $C^{*}$-dynamical system structure in terms of Bogoliubov automorphisms on the CAR algebra of observables $\mathcal{O}$ over the corresponding one-particle Hilbert space $\mathfrak{h}$. Recall that a $C^{*}$-dynamical system is a pair $(\mathcal{O}, \tau)$ with

$$
\mathbb{R} \ni t \mapsto \tau^{t} \in \operatorname{Aut}(\mathcal{O}),
$$

where $\mathcal{O}$ is a $C^{*}$ algebra and $\tau^{t}$ a strongly continuous group of $*$-automorphism of $\mathcal{O}$ (for more information on the algebraic approach to open quantum systems, see, for example, Aschbacher et al. [7]). Moreover, let us denote the states on $\mathcal{O}$ by $\mathcal{E}(\mathcal{O})$ and recall that a state $\omega \in \mathcal{E}(\mathcal{O})$ is called a (gauge invariant) quasifree state with density $\rho \in \mathcal{L}(\mathfrak{h})$ satisfying $0 \leq \rho \leq 1$ if, for all $p, q \in \mathbb{N}$ and all $f_{i}, g_{j} \in \mathfrak{h}$ with $i, j \in \mathbb{N}$, we have

$$
\omega\left(a^{*}\left(f_{p}\right) \ldots a^{*}\left(f_{1}\right) a\left(g_{1}\right) \ldots a\left(g_{q}\right)\right)=\delta_{p q} \operatorname{det}\left(\left[\left(g_{i}, \rho f_{j}\right)\right]_{i, j=1}^{p}\right),
$$

where $a^{*}(f), a(f) \in \mathcal{L}(\mathfrak{F}(\mathfrak{h}))$ with $f \in \mathfrak{h}$ stand for the usual creation and annihilation operators on the fermionic Fock space $\mathfrak{F}(\mathfrak{h})$ over the one-particle Hilbert space $\mathfrak{h}$. Here, we used the notation $\mathcal{L}(\mathcal{G}, \mathcal{H})$ for the bounded linear operators from some Hilbert space $\mathcal{G}$ into some Hilbert space $\mathcal{H}\left(\right.$ with $\mathcal{L}(\mathcal{H}):=\mathcal{L}(\mathcal{H}, \mathcal{H})$ ), and $\delta_{a b}$ denotes the usual Kronecker 
symbol. In the following, we will also use the notations $\mathbb{N}_{0}:=\{0\} \cup \mathbb{N}$ and $\operatorname{Re}(A):=$ $\left(A+A^{*}\right) / 2$ and $\operatorname{Im}(A):=\left(A-A^{*}\right) /(21)$ for all $A \in \mathcal{L}(\mathcal{H})$, and $\mathcal{L}^{0}(\mathcal{H})$ and $\mathcal{L}^{1}(\mathcal{H})$ stand for the finite rank operators and the trace class operators, respectively. Moreover, $\mathrm{d} \Gamma(A)$ is the usual second quantization on the fermionic Fock space.

Definition 1 (Quasifree setting) The ingredients for this setting are specified as follows.

(a) Observable algebra

Let $n \in \mathbb{N}_{0}$. The sample and the reservoir configuration spaces are defined by

$$
\begin{aligned}
& \mathbb{Z}_{\mathcal{S}}:=\{x \in \mathbb{Z}|| x \mid \leq n\}, \\
& \mathbb{Z}_{\mathcal{R}}:=\{x \in \mathbb{Z}|| x \mid \geq n+1\},
\end{aligned}
$$

whereas the subreservoir spaces are given by

$$
\begin{aligned}
& \mathbb{Z}_{L}:=\{x \in \mathbb{Z} \mid x \leq-(n+1)\}, \\
& \mathbb{Z}_{R}:=\{x \in \mathbb{Z} \mid x \geq n+1\},
\end{aligned}
$$

see Figure 1 . The observable algebra is then defined to be the CAR algebra

$$
\mathcal{O}:=\mathfrak{A}\left(\mathfrak{h}_{\mathcal{S}} \oplus \mathfrak{h}_{\mathcal{R}}\right)
$$

where the one-particle Hilbert space consists of the sample and the reservoir space

$$
\begin{aligned}
\mathfrak{h}_{\mathcal{S}} & :=\ell^{2}\left(\mathbb{Z}_{\mathcal{S}}\right), \\
\mathfrak{h}_{\mathcal{R}} & :=\ell^{2}\left(\mathbb{Z}_{\mathcal{R}}\right),
\end{aligned}
$$

and the dimension of the sample Hilbert space is denoted by $n_{\mathcal{S}}:=\operatorname{dim}\left(\mathfrak{h}_{\mathcal{S}}\right)=2 n+1$. Moreover, the one-particle Hilbert spaces of the subreservoirs are given by

$$
\begin{aligned}
\mathfrak{h}_{L} & :=\ell^{2}\left(\mathbb{Z}_{L}\right), \\
\mathfrak{h}_{R} & :=\ell^{2}\left(\mathbb{Z}_{R}\right) .
\end{aligned}
$$

For $a \in\{\mathcal{S}, \mathcal{R}, L, R\}$, using the map $i_{a} \in \mathcal{L}\left(\mathfrak{h}_{a}, \mathfrak{h}\right)$ defined, for all $f \in \mathfrak{h}_{a}$, by $i_{a} f(x):=$ $f(x)$ if $x \in \mathbb{Z}_{a}$ and $i_{a} f(x):=0$ if $x \in \mathbb{Z} \backslash \mathbb{Z}_{a}$, the total one-particle Hilbert space is naturally identified with

$$
\mathfrak{h}:=\ell^{2}(\mathbb{Z})
$$

through $f \mapsto i_{\mathcal{S}}^{*} f \oplus i_{\mathcal{R}}^{*} f$ for all $f \in \mathfrak{h}$. Analogously, $\mathfrak{h}_{\mathcal{R}}$ is identified with $\mathfrak{h}_{L} \oplus \mathfrak{h}_{R}$ through $f \mapsto i_{L}^{*} i_{\mathcal{R}} f \oplus i_{R}^{*} i_{\mathcal{R}} f$ for all $f \in \mathfrak{h}_{\mathcal{R}}$.

(b) Dynamics

Let $\lambda \in \mathbb{R}$. The one-particle Hamiltonians $h, h_{\lambda} \in \mathcal{L}(\mathfrak{h})$ are defined by

$$
\begin{aligned}
h & :=\operatorname{Re}[u], \\
h_{\lambda} & :=h+(\lambda-1) v \\
& =h_{0}+\lambda v,
\end{aligned}
$$


where the right translation $u \in \mathcal{L}(\mathfrak{h})$ is defined by $(u f)(x):=f(x-1)$ for all $f \in \mathfrak{h}$ and all $x \in \mathbb{Z}$. The operator $h_{1}=h$ is called the $X Y$ Hamiltonian, $h_{0}$ the decoupled Hamiltonian, and $h_{\lambda}$ with $\lambda \neq 0$ the coupled Hamiltonian. Moreover, the operator $v \in \mathcal{L}^{0}(\mathfrak{h})$, called the bond perturbation, is defined by

$$
\begin{aligned}
v & :=\sum_{a \in\{L, R\}} v_{a}, \\
v_{a} & :=\operatorname{Re}\left[\left(\delta_{\mathcal{S}, a}, \cdot\right) \delta_{\mathcal{R}, a}\right],
\end{aligned}
$$

where the coupling functions are specified by

$$
\delta_{\mathcal{S}, L}:=\delta_{-n}, \quad \delta_{\mathcal{S}, R}:=\delta_{n}, \quad \delta_{\mathcal{R}, L}:=\delta_{-(n+1)}, \quad \delta_{\mathcal{R}, R}:=\delta_{n+1},
$$

and $\delta_{x} \in \mathfrak{h}$ with $x \in \mathbb{Z}$ is given by $\delta_{x}(y):=\delta_{x y}$ for all $y \in \mathbb{Z}$. Moreover, for $a \in$ $\{\mathcal{S}, \mathcal{R}, L, R\}$, the one-particle Hamiltonians $h_{a} \in \mathcal{L}\left(\mathfrak{h}_{a}\right)$ are defined by

$$
h_{a}:=i_{a}^{*} h i_{a} .
$$

The second quantized Hamiltonians on the fermionic Fock space $\mathfrak{F}(\mathfrak{h})$ are given by

$$
\begin{aligned}
H & :=\mathrm{d} \Gamma(h), \\
V & :=\sum_{a \in\{L, R\}} V_{a}, \\
V_{a} & :=\mathrm{d} \Gamma\left(v_{a}\right), \\
H_{\lambda} & :=H+(\lambda-1) V \\
& =H_{0}+\lambda V,
\end{aligned}
$$

where $H$ is unbounded and $V_{a} \in \mathcal{O}$ is a local perturbation. Finally, the dynamics $\tau_{\lambda}^{t} \in \operatorname{Aut}(\mathcal{O})$ with $t, \lambda \in \mathbb{R}$ are defined, for all $A \in \mathcal{O}$, by

$$
\tau_{\lambda}^{t}(A):=\mathrm{e}^{\mathrm{i} t H_{\lambda}} A \mathrm{e}^{-1 t H_{\lambda}} .
$$

(c) Initial state

The initial state $\omega \in \mathcal{E}(\mathcal{O})$ is defined to be quasifree with density $\rho \in \mathcal{L}(\mathfrak{h})$ defined by

$$
\rho:=i_{\mathcal{S}} \rho_{\mathcal{S}} i_{\mathcal{S}}^{*}+i_{\mathcal{R}} \rho_{\mathcal{R}} i_{\mathcal{R}}^{*},
$$

where $\rho_{\mathcal{S}} \in \mathcal{L}\left(\mathfrak{h}_{\mathcal{S}}\right)$ and $\rho_{\mathcal{R}} \in \mathcal{L}\left(\mathfrak{h}_{\mathcal{R}}\right)$ are given by

$$
\begin{aligned}
\rho_{\mathcal{S}} & :=\varrho_{\beta_{\mathcal{S}}}\left(h_{\mathcal{S}}\right), \\
\rho_{\mathcal{R}} & :=\sum_{a \in\{L, R\}} i_{\mathcal{R}}^{*} i_{a} \varrho_{\beta_{a}}\left(h_{a}\right) i_{a}^{*} i_{\mathcal{R}} .
\end{aligned}
$$

Here, for any $\alpha \in \mathbb{R}$, the Planck density function $\varrho_{\alpha}: \mathbb{R} \rightarrow \mathbb{R}$ is defined by

$$
\varrho_{\alpha}(e):=\left(1+\mathrm{e}^{\alpha e}\right)^{-1},
$$


Pour fixer les idées, we will always assume that the inverse temperatures of the sample and the reservoirs satisfy

$$
\beta_{\mathcal{S}}=0, \quad 0<\beta_{L} \leq \beta_{R}<\infty
$$

and we set $\beta:=\left(\beta_{R}+\beta_{L}\right) / 2$ and $\delta:=\left(\beta_{R}-\beta_{L}\right) / 2$.

Remark 2 As discussed in the introduction, this model has its origin in the $X Y$ spin chain whose formal Hamiltonian reads

$$
H_{X Y}=-\frac{1}{4} \sum_{x \in \mathbb{Z}}\left\{(1+\gamma) \sigma_{1}^{(x)} \sigma_{1}^{(x+1)}+(1-\gamma) \sigma_{2}^{(x)} \sigma_{2}^{(x+1)}+2 \mu \sigma_{3}^{(x)}\right\},
$$

where $\gamma \in(-1,1)$ denotes the anisotropy, $\mu \in \mathbb{R}$ the external magnetic field, and the Pauli basis of $\mathbb{C}^{2 \times 2}$ is given by

$$
\sigma_{0}=\left[\begin{array}{ll}
1 & 0 \\
0 & 1
\end{array}\right], \quad \sigma_{1}=\left[\begin{array}{ll}
0 & 1 \\
1 & 0
\end{array}\right], \quad \sigma_{2}=\left[\begin{array}{cc}
0 & -1 \\
1 & 0
\end{array}\right], \quad \sigma_{3}=\left[\begin{array}{cc}
1 & 0 \\
0 & -1
\end{array}\right] .
$$

Namely, under the Araki-Jordan-Wigner transformation (see, for example, Araki [4]), the Hamiltonian from (13) corresponds to the case of the so-called isotropic XY chain (or XX chain) without external magnetic field, i.e. to the case where $\gamma=0$ and $\mu=0$. In order to treat the anisotropic case $\gamma \neq 0$, one often uses the so-called selfdual quasifree setup introduced and developed in Araki [2, 3]. There, one works in the doubled oneparticle Hilbert space $\mathfrak{h}^{\oplus 2}$ and the generator of the truly anisotropic $X Y$ dynamics has nontrivial off-diagonal blocks on $\mathfrak{h}^{\oplus 2}$ (which vanish for $\gamma=0$ ). In many respects, the truly anisotropic XY model is substantially more complicated than the isotropic one (this is true a fortiori if a magnetic field is added whose contribution to the generator acts diagonally on $\mathfrak{h}^{\oplus 2}$ though). In the following, every once in a while, we will make a remark on the corresponding issue for the anisotropic case.

Remark 3 The Hamiltonian $h_{\lambda}$ for $\lambda=0$ does not couple the different subsystems to each other, i.e. we have $h_{0}=\sum_{a \in\{\mathcal{S}, L, R\}} i_{a} h_{a} i_{a}^{*}$.

In order to construct a NESS, we use the following definition due to Ruelle [20]. If not specified otherwise, it will always be assumed that $n \in \mathbb{N}_{0}$ and $\lambda \in \mathbb{R}$.

Definition 4 (NESS) A NESS associated with the $C^{*}$-dynamical system $\left(\mathcal{O}, \tau_{\lambda}^{t}\right)$ and the initial state $\omega \in \mathcal{E}(\mathcal{O})$ is a weak-* limit point for $T \rightarrow \infty$ of the net

$$
\left\{\frac{1}{T} \int_{0}^{T} \mathrm{~d} t \omega \circ \tau_{\lambda}^{t} \mid T>0\right\} .
$$

Such a NESS is denoted by $\omega_{\lambda,+} \in \mathcal{E}(\mathcal{O})$. 
If the coupled time evolution is the XY dynamics (i.e. if $\lambda=1$ ), the unique quasifree NESS in the fully anisotropic $X Y$ model with magnetic field has been constructed in Aschbacher and Pillet [6]. In order to state the corresponding theorem, we switch to the momentum space representation by using the Fourier transformation $\mathfrak{f}: \mathfrak{h} \rightarrow \widehat{\mathfrak{h}}$, where

$$
\widehat{\mathfrak{h}}:=L^{2}\left([-\pi, \pi] ; \frac{\mathrm{d} k}{2 \pi}\right),
$$

and $\mathfrak{f}$ is defined with the sign convention $\widehat{f}(k):=(\mathfrak{f} f)(k):=\sum_{x \in \mathbb{Z}} f(x) \mathrm{e}^{\mathrm{i} k x}$. Moreover, we also use the notation $\widehat{A}:=\mathfrak{f} A \mathfrak{f}^{*}$ for all $A \in \mathcal{L}(\mathfrak{h})$. In the following, for any selfadjoint $A, B \in \mathcal{L}(\mathfrak{h})$, we denote by $w(A, B) \in \mathcal{L}(\mathfrak{h})$ the wave operator

$$
w(A, B):=\mathrm{s}-\lim _{t \rightarrow \infty} \mathrm{e}^{-\mathrm{i} t A} \mathrm{e}^{\mathrm{i} t B} 1_{\mathrm{ac}}(B),
$$

where $1_{\mathrm{ac}}(B)$ is the spectral projection onto the absolutely continuous subspace of $B$.

Theorem 5 (XY NESS) There exists a unique quasifree NESS $\omega_{1,+} \in \mathcal{E}(\mathcal{O})$ associated with the $C^{*}$-dynamical system $\left(\mathcal{O}, \tau_{1}^{t}\right)$ and the initial state $\omega \in \mathcal{E}(\mathcal{O})$. Moreover, its density $\rho_{1,+} \in \mathcal{L}(\mathfrak{h})$ has the form

$$
\begin{aligned}
\rho_{1,+} & =w^{*}\left(h_{0}, h\right) \rho w\left(h_{0}, h\right) \\
& =\left(1+\mathrm{e}^{\beta h-\delta d}\right)^{-1},
\end{aligned}
$$

where, in momentum space, $\widehat{h}, \widehat{d} \in \mathcal{L}(\widehat{\mathfrak{h}})$ are the multiplication operators acting, for all $\varphi \in \widehat{\mathfrak{h}}$ and all $k \in(-\pi, \pi]$, as

$$
\begin{aligned}
\widehat{h} \varphi(k) & =\epsilon(k) \varphi(k), \\
\widehat{d} \varphi(k) & =\operatorname{sign}\left(\epsilon^{\prime}(k)\right) \widehat{h} \varphi(k),
\end{aligned}
$$

and the dispersion relation $\epsilon: \mathbb{R} \rightarrow \mathbb{R}$ is given by

$$
\epsilon(k):=\cos (k) .
$$

Proof. See Aschbacher and Pillet [6].

In the following, we denote by $1_{e}\left(h_{\lambda}\right) \in \mathcal{L}(\mathfrak{h})$ the usual spectral projection onto the eigenspace of $h_{\lambda}$ corresponding to the eigenvalue $e \in \operatorname{spec}_{\mathrm{pp}}\left(h_{\lambda}\right)$. Moreover, $\operatorname{spec}_{\mathrm{sc}}\left(h_{\lambda}\right)$ is the singular continuous spectrum of $h_{\lambda}$. We then have the following result.

Theorem 6 (Microscopic NESS) There exists a unique NESS $\omega_{\lambda,+} \in \mathcal{E}(\mathcal{O})$ associated with the $C^{*}$-dynamical system $\left(\mathcal{O}, \tau_{\lambda}^{t}\right)$ and the initial state $\omega \in \mathcal{E}(\mathcal{O})$. Moreover, its density $\rho_{\lambda,+} \in \mathcal{L}(\mathfrak{h})$ has the form

$$
\rho_{\lambda,+}=w^{*}\left(h_{0}, h_{\lambda}\right) \rho w\left(h_{0}, h_{\lambda}\right)+\sum_{e \in \operatorname{spec}_{\mathrm{pp}}\left(h_{\lambda}\right)} 1_{e}\left(h_{\lambda}\right) \rho 1_{e}\left(h_{\lambda}\right) .
$$


Proof. Since $h_{\lambda} \in \mathcal{L}(\mathfrak{h}), h_{\lambda}-h_{0} \in \mathcal{L}^{0}(\mathfrak{h})$, and $\operatorname{spec}_{\mathrm{sc}}\left(h_{\lambda}\right)=\emptyset$ due to Lemma 30 of Appendix A, we can use Aschbacher et al. [8] which yields the assertion.

Remark 7 As given in Lemma 30 of Appendix A, the pure point component in (38) is absent if $0<|\lambda| \leq 1$ (see also Jakšić et al. [17]).

We next turn to the energy current observable and its NESS expectation.

Definition 8 (Energy current) The observable $\Phi_{\lambda, a} \in \mathcal{O}$ with $a \in\{L, R\}$ describing the energy current flowing from reservoir a into the sample is defined by

$$
\Phi_{\lambda, a}:=\mathrm{d} \Gamma\left(\varphi_{\lambda, a}\right),
$$

where the one-particle energy current observable $\varphi_{\lambda, a} \in \mathcal{L}^{0}(\mathfrak{h})$ is given by

$$
\varphi_{\lambda, a}:=-\left.\frac{\mathrm{d}}{\mathrm{d} t}\right|_{t=0} \mathrm{e}^{\mathrm{i} t h_{\lambda}} i_{a} h_{a} i_{a}^{*} \mathrm{e}^{-1 t h_{\lambda}} .
$$

Moreover, its NESS expectation value is denoted by

$$
J_{\lambda, a}:=\omega_{\lambda,+}\left(\Phi_{\lambda, a}\right) .
$$

Let us next turn to the structure of the NESS current. The following proposition shows, on one hand, that the expectation value is independent of the pure point component of the NESS density. On the other hand, it implies that we can later proceed to its computation by exploiting the known density of the XY NESS and the purely absolutely continuous nature of the $\mathrm{XY}$ Hamiltonian in the construction of the wave operator. The commutator of $A, B \in \mathcal{L}(\mathcal{H})$ is denoted by $[A, B]:=A B-B A$.

Proposition 9 (Energy current structure) For $a \in\{L, R\}$, we have

$$
J_{\lambda, a}=\operatorname{tr}\left(w^{*}\left(h, h_{\lambda}\right) \rho_{1,+} w\left(h, h_{\lambda}\right) \varphi_{\lambda, a}\right) .
$$

Proof. Since $\varphi_{\lambda, a} \in \mathcal{L}^{0}(\mathfrak{h})$ and using the form (38) of the NESS density, we can write

$$
\begin{aligned}
J_{\lambda, a} & =\operatorname{tr}\left(\rho_{\lambda,+} \varphi_{\lambda, a}\right) \\
& =\operatorname{tr}\left(w^{*}\left(h_{0}, h_{\lambda}\right) \rho w\left(h_{0}, h_{\lambda}\right) \varphi_{\lambda, a}\right)+\sum_{e \in \operatorname{spec}_{\mathrm{pp}}\left(h_{\lambda}\right)} \operatorname{tr}\left(1_{e}\left(h_{\lambda}\right) \rho 1_{e}\left(h_{\lambda}\right) \varphi_{\lambda, a}\right) .
\end{aligned}
$$

The independence of the current of the pure point component of the NESS density now follows as in Aschbacher et al. [8] from the observation that, since the one-particle energy current observable from (40) has the form of a commutator, namely $\varphi_{\lambda, a}=-1\left[h_{\lambda}, i_{a} h_{a} i_{a}^{*}\right]$, we have, for all $e \in \operatorname{spec}_{\mathrm{pp}}\left(h_{\lambda}\right)$, that

$$
1_{e}\left(h_{\lambda}\right) \varphi_{\lambda, a} 1_{e}\left(h_{\lambda}\right)=0 .
$$


Applying the chain rule $w\left(h_{0}, h_{\lambda}\right)=w\left(h_{0}, h\right) w\left(h, h_{\lambda}\right)$ to the wave operators in (43) (which is applicable since the perturbations are trace class) and using (34), we get

$$
w^{*}\left(h_{0}, h_{\lambda}\right) \rho w\left(h_{0}, h_{\lambda}\right)=w^{*}\left(h, h_{\lambda}\right) \rho_{1,+} w\left(h, h_{\lambda}\right) .
$$

This is the assertion.

Remark 10 Note that, due to $\varphi_{\lambda, a}=-1\left[h_{\lambda}, i_{a} h_{a} i_{a}^{*}\right]=-1 \lambda\left[v_{a}, i_{a} h_{a} i_{a}^{*}\right] \in \mathcal{L}^{0}(\mathfrak{h})$, we have $\sum_{a \in\{L, R\}} \varphi_{\lambda, a}=1\left[h_{\lambda}, i_{\mathcal{S}} h_{\mathcal{S}} i_{\mathcal{S}}^{*}+\lambda v\right]$, where $i_{\mathcal{S}} h_{\mathcal{S}} i_{\mathcal{S}}^{*}+\lambda v \in \mathcal{L}^{0}(\mathfrak{h})$. Hence, $\mathrm{d} \Gamma\left(i_{\mathcal{S}} h_{\mathcal{S}} i_{\mathcal{S}}^{*}+\lambda v\right) \in \mathcal{O}$, and

$$
\sum_{a \in\{L, R\}} \Phi_{\lambda, a}=\left.\frac{\mathrm{d}}{\mathrm{d} t}\right|_{t=0} \tau_{\lambda}^{t}\left(\mathrm{~d} \Gamma\left(i_{\mathcal{S}} h_{\mathcal{S}} i_{\mathcal{S}}^{*}+\lambda v\right)\right) .
$$

Since Definition 4 implies that the NESS is invariant under the corresponding $C^{*}$ dynamics, i.e. since $\omega_{\lambda,+} \circ \tau_{\lambda}^{t}=\omega_{\lambda,+}$ for all $\lambda, t \in \mathbb{R}$, we get the first law of thermodynamics of the microscopic regime,

$$
\sum_{a \in\{L, R\}} J_{\lambda, a}=0 .
$$

Therefore, we can restrict ourselves to study the objects from Definition 8 for $a=L$, and, for this case, we drop the index $L$ in the notation.

We now arrive at the first of our main theorems. For any coupling strength and any size of the sample, it yields an explicit expression for the NESS energy current and, thus, for the microscopic entropy production (see, for example, Aschbacher et al. [7]),

$$
\mathrm{Ep}_{\lambda}:=-\sum_{a \in\{L, R\}} \beta_{a} J_{\lambda, a}=2 \delta J_{\lambda} .
$$

Of course, for $\lambda=0$, we have $\mathrm{Ep}_{0}=0$. If $\lambda \neq 0$, we have the following result.

Theorem 11 (Microscopic second law of thermodynamics) For $\lambda \neq 0$, the microscopic entropy production is given by the absolutely convergent integral

$$
\mathrm{Ep}_{\lambda}=\delta \lambda^{4} \int_{-\pi}^{\pi} \frac{\mathrm{d} k}{2 \pi} \frac{S(|\epsilon(k)|)}{Q_{\lambda}(|\epsilon(k)|)},
$$

where the functions $S:[-1,1] \rightarrow \mathbb{R}$ and $Q_{\lambda}:(-1,1) \rightarrow \mathbb{R}$ are defined by

$$
\begin{aligned}
S(e) & :=e\left(1-e^{2}\right)^{1 / 2}\left[\varrho_{\beta_{L}}(e)-\varrho_{\beta_{R}}(e)\right], \\
Q_{\lambda} & :=\left|\left(1-E^{2}\right)^{-2}\left[\left(1-\lambda^{2} E^{2}\right)^{2}-\left(1-\lambda^{2}\right)^{2} E^{2\left(n_{\mathcal{S}}+1\right)}\right]\right|^{2},
\end{aligned}
$$

and $E(e):=e+1\left(1-e^{2}\right)^{1 / 2}$. Thus, if the system is truly out of equilibrium, i.e. if $\delta \neq 0$, the microscopic entropy production is strictly positive and the energy current is flowing through the sample from the hotter to the colder reservoir. 
Remark 12 We can rewrite the microscopic entropy production in the form

$$
\operatorname{Ep}_{\lambda}=\frac{\delta \lambda^{4}}{2} \int_{-\pi}^{\pi} \frac{\mathrm{d} k}{2 \pi} \frac{\epsilon(k)\left|\epsilon^{\prime}(k)\right|}{Q_{\lambda}(\epsilon(k))} \frac{\operatorname{sh}[\delta \epsilon(k)]}{\operatorname{ch}^{2}\left[\frac{\beta}{2} \epsilon(k)\right]+\operatorname{sh}^{2}\left[\frac{\delta}{2} \epsilon(k)\right]},
$$

where we used the convenient identity $2\left[\left(1+\mathrm{e}^{x}\right)^{-1}-\left(1+\mathrm{e}^{y}\right)^{-1}\right]=\operatorname{sh}[(y-x) / 2] /\left(\operatorname{ch}^{2}[(x+\right.$ $\left.y) / 4]+\operatorname{sh}^{2}[(x-y) / 4]\right)$ for $x, y \in \mathbb{R}$. For $\lambda=1$, we have $Q_{1}=1$ and, hence, we recover the expression found in Aschbacher and Pillet [6] (which, in addition, is also independent of the sample size).

Proof. In order to analyze the NESS current, we start from (42) and determine its ingredients. For convenience, we will work with the objects for $a=R$ in this proof. First, using (40), we can write the one-particle energy current observable as

$$
\varphi_{\lambda, R}=\frac{\lambda}{2} \operatorname{Im}\left[\left(\delta_{n}, \cdot\right) \delta_{n+2}\right]
$$

Plugging (53) into (42), we get

$$
J_{\lambda, R}=\frac{\lambda}{2} \operatorname{Im}\left[F_{\lambda}(n, n+2)\right]
$$

where, for all $\lambda \in \mathbb{R}$, the function $F_{\lambda}: \mathbb{Z}^{2} \rightarrow \mathbb{C}$ is defined by

$$
F_{\lambda}(x, y):=\left(w\left(h, h_{\lambda}\right) \delta_{x}, \rho_{1,+} w\left(h, h_{\lambda}\right) \delta_{y}\right) .
$$

In order to compute this function and, in particular, the wave operator appearing in it, we switch to the energy space of the XY Hamiltonian (i.e. to the space diagonalizing $h$ ) given in Lemma 27 of Appendix A by

$$
\widetilde{\mathfrak{h}}=L^{2}\left([-1,1], \mathbb{C}^{2} ; \mathrm{d} e\right)
$$

In this representation, the action of the wave operator on the completely localized wave functions $\delta_{x} \in \mathfrak{h}$ with $x \in \mathbb{Z}$ reads

$$
\widetilde{w}\left(h, h_{\lambda}\right) \widetilde{\delta}_{x}=\widetilde{\delta}_{x}-\frac{\lambda-1}{2} \sum_{i, j=1}^{4} \Sigma_{\lambda, i j}^{-1}(\cdot-10) \varrho_{\delta_{j}^{1}, \delta_{x}}(\cdot-10) \widetilde{\delta}_{i}^{2}
$$

where $\Sigma_{\lambda}(e-10) \in \mathbb{C}^{4 \times 4}$ and $\varrho_{\delta_{j}^{1}, \delta_{x}}(e-10) \in \mathbb{C}$ are the boundary values of the interaction matrix and of the $X Y$ resolvent amplitudes, respectively, and $\delta_{i}^{1}, \delta_{i}^{2} \in \mathfrak{h}$ are the coupling functions, see Proposition 35 of Appendix B. Plugging (57) into (55), we get

$$
F_{\lambda}(x, y)=\int_{-1}^{1} \mathrm{~d} e S_{x, y}^{(0)}(e)+\sum_{i=1}^{2}\left(\frac{1-\lambda}{2}\right)^{i} \int_{-1}^{1} \mathrm{~d} e S_{\lambda, x, y}^{(i)}(e)
$$


where, for all $\lambda \in \mathbb{R}, x, y \in \mathbb{Z}$, and $i=1,2$, the functions $S_{x, y}^{(0)}, S_{\lambda, x, y}^{(i)}:(-1,1) \rightarrow \mathbb{C}$ read

$$
\begin{aligned}
S_{x, y}^{(0)} & :=\left\langle\widetilde{\delta}_{x}, \widetilde{\rho}_{1,+} \widetilde{\delta}_{y}\right\rangle_{2}, \\
S_{\lambda, x, y}^{(1)} & :=\left\langle\xi_{x}, \Sigma_{\lambda}^{-1}(\cdot-10) \eta_{y}\right\rangle_{4}+\left\langle\Sigma_{\lambda}^{-1}(\cdot-10) \eta_{x}, \xi_{y}\right\rangle_{4}, \\
S_{\lambda, x, y}^{(2)} & :=\left\langle\Sigma_{\lambda}^{-1}(\cdot-10) \eta_{x}, \Theta \Sigma_{\lambda}^{-1}(\cdot-10) \eta_{y}\right\rangle_{4} .
\end{aligned}
$$

Here, for all $x \in \mathbb{Z}$, the vector-valued functions $\xi_{x}, \eta_{x}:(-1,1) \rightarrow \mathbb{C}^{4}$ and the matrix-valued function $\Theta:(-1,1) \rightarrow \mathbb{C}^{4 \times 4}$ are defined, for $i, j=1, \ldots, 4$, by

$$
\begin{aligned}
& \xi_{x, i}:=\left\langle\widetilde{\delta}_{i}^{2}, \widetilde{\rho}_{1,+} \widetilde{\delta}_{x}\right\rangle_{2}, \\
& \eta_{x, i}:=\varrho_{\delta_{i}^{1}, \delta_{x}}(\cdot-10), \\
& \Theta_{i j}:=\left\langle\widetilde{\delta}_{i}^{2}, \widetilde{\rho}_{1,+} \widetilde{\delta}_{j}^{2}\right\rangle_{2},
\end{aligned}
$$

where $\langle\cdot, \cdot\rangle_{d}$ stands for the Euclidean scalar product in $\mathbb{C}^{d}$. We next specialize to the case at hand, namely to $x=n$ and $y=n+2$. For this case, the ingredients of (59)-(61) are computed in Lemma 39 of Appendix B. Plugging these expressions into (58), we get

$$
F_{\lambda}(n, n+2)=F_{1}(n, n+2)+\int_{-1}^{1} \mathrm{~d} e \frac{\sum_{i=0}^{8} p_{i}(e) \lambda^{i}}{Q_{\lambda}(e)},
$$

where the function $Q_{\lambda}:(-1,1) \rightarrow \mathbb{R}$, defined by $Q_{\lambda}:=\mid \operatorname{det}\left(\left.\Sigma_{\lambda}(\cdot-10)\right|^{2}\right.$, has the expansion $Q_{\lambda}=\sum_{i=0}^{4} q_{2 i} \lambda^{2 i}$, and the coefficient functions $p_{i}, q_{i}:(-1,1) \rightarrow \mathbb{C}$ are given in Lemma 40 of Appendix $B$. Subtracting $F_{0}(n, n+2)=0$ from (65) (where the latter follows from Lemma 40, see also (55), we can write

$$
F_{\lambda}(n, n+2)=\int_{-1}^{1} \mathrm{~d} e \frac{\sum_{i=0}^{3} p_{2 i+1}(e) \lambda^{2 i+1}}{Q_{\lambda}(e)},
$$

where we used that $q_{0} p_{2 i}-p_{0} q_{2 i}=0$ for $i=1, \ldots, 4$, see Lemma 40 of Appendix B. Transforming the coordinates as $e=\epsilon(k)$ for $k \in[0, \pi]$ and using that $\operatorname{Im}\left[p_{2 i+1}(\epsilon(k))\right]=0$ for $i=0,2,3$ and $\operatorname{Im}\left[p_{3}(\epsilon(k))\right]=-\epsilon(k)\left[\varrho_{\beta_{L}}(\epsilon(k))-\varrho_{\beta_{R}}(\epsilon(k))\right] / \pi$ from Lemma 40, we get (49). Finally, due to Lemma 41 in Appendix $\mathrm{B}$, the integral in (49) is absolutely convergent, and since the numerator and the denominator are even functions in $e$, we arrive at the assertion.

\section{Leading order microscopic regime}

In this section, we determine the leading order contribution to the microscopic entropy production from Theorem 11 for small bond coupling $\lambda$. It has the following form. 


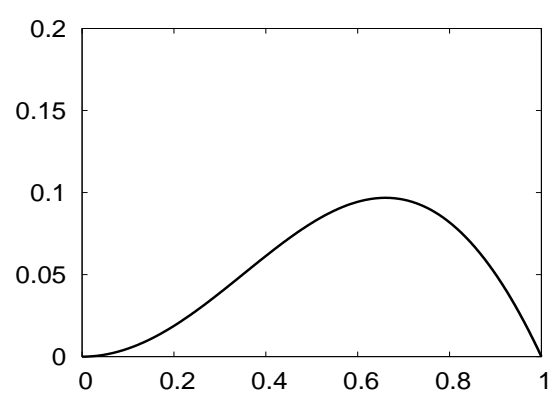

Figure 2: The function $S_{0}$ on $[0,1]$ for $\beta_{L}=1$ and $\beta_{R}=2$.

Theorem 13 (Leading order contribution) For $\lambda \rightarrow 0$, the microscopic entropy production has the expansion

$$
\operatorname{Ep}_{\lambda}=\operatorname{Ep} \lambda^{2}+\mathcal{O}\left(\lambda^{4}\right)
$$

where the second order contribution has the form

$$
\operatorname{Ep}:=\frac{2 \delta}{n_{\mathcal{S}}+1} \sum_{i=1}^{n_{\mathcal{S}}} S_{0}\left(\epsilon\left(k_{i}\right)\right),
$$

and the function $S_{0}:[-1,1] \rightarrow \mathbb{R}$ (see Figure 2) and the momenta $k_{i}$ for $i=1, \ldots, n_{\mathcal{S}}$ read

$$
\begin{aligned}
S_{0}(e) & :=e\left(1-e^{2}\right)^{3 / 2}\left[\varrho_{\beta_{L}}(e)-\varrho_{\beta_{R}}(e)\right], \\
k_{i} & :=\frac{i \pi}{n_{\mathcal{S}}+1} .
\end{aligned}
$$

Remark 14 Note that $\epsilon\left(k_{n+1+i}\right)=-\epsilon\left(k_{n+1-i}\right)$ for $i=1, \ldots, n$ and that $S_{0}$ is an even function. Hence, for $n>0$, we can further simplify (68) as $\mathrm{Ep}=2 \delta /(n+1) \sum_{i=1}^{n} S_{0}\left(\epsilon\left(k_{i}\right)\right)$.

Remark 15 It follows from $\epsilon\left(k_{n+1}\right)=0$ that, for $n=0$, we have $\mathrm{Ep}=0$. Hence, in this case, the entropy production from Theorem 11 is carried by higher orders than the second one. On the other hand, since $S_{0} \circ \epsilon \in C\left([-\pi, \pi] ; \frac{\mathrm{d} k}{2 \pi}\right)$ is an even function, we get with the identity from Remark 12 that

$$
\lim _{n \rightarrow \infty} \operatorname{Ep}=\delta \int_{-\pi}^{\pi} \frac{\mathrm{d} k}{2 \pi} \epsilon(k)\left|\epsilon^{\prime}(k)\right|^{3} \frac{\operatorname{sh}[\delta \epsilon(k)]}{\operatorname{ch}^{2}\left[\frac{\beta}{2} \epsilon(k)\right]+\operatorname{sh}^{2}\left[\frac{\delta}{2} \epsilon(k)\right]} .
$$

Let us now turn to the proof of Theorem 13 .

Proof. In order to extract the second order contribution to the NESS current, we determine the limit $\lambda \rightarrow 0$ of $J_{\lambda} / \lambda^{2}$ with the help of a Sokhotski-Plemelj type argument. For this 
purpose, we rewrite the quotient in (49) as $S / Q_{\lambda}=N / D_{\lambda}$, where $N, D_{\lambda}: \mathbb{R} \rightarrow \mathbb{R}$ read

$$
\begin{aligned}
N & :=4\left(1-\epsilon^{2}\right)^{2} S(|\epsilon|), \\
D_{\lambda} & :=L_{\lambda}+\lambda^{4} R_{\lambda},
\end{aligned}
$$

and the functions $L_{\lambda}, R_{\lambda}: \mathbb{R} \rightarrow \mathbb{R}$ are given by

$$
\begin{aligned}
& L_{\lambda}:=\sum_{i \in\{0,2\}} d_{i} \lambda^{i}+d_{4^{\prime}} \lambda^{4}, \\
& R_{\lambda}:=\sum_{i \in\{4,6,8\}} d_{i} \lambda^{4-i}-d_{4^{\prime}} .
\end{aligned}
$$

Moreover, the coefficients $d_{i}: \mathbb{R} \rightarrow \mathbb{R}$ for $i=0,2,4,4^{\prime}, 6,8$ have the form

$$
\begin{aligned}
d_{0} & =\sigma_{n_{0}}^{2} \\
d_{2} & =-4 \sigma_{n_{0}} \sigma_{n_{1}} \epsilon \\
d_{4} & =2 \sigma_{n_{0}} \sigma_{n_{2}} \epsilon_{2}+4 \sigma_{n_{1}}^{2} \\
d_{4^{\prime}} & :=4 \sigma_{n_{1}}^{2} \epsilon^{2} \\
d_{6} & =-4 \sigma_{n_{1}} \sigma_{n_{2}} \epsilon \\
d_{8} & =\sigma_{n_{2}}^{2}
\end{aligned}
$$

where, for all $\alpha \in \mathbb{R}$, we used the notation $\sigma_{\alpha}(k):=\sin (\alpha k), \sigma:=\sigma_{1}$, and $\epsilon_{\alpha}(k):=\epsilon(\alpha k)$ for all $k \in \mathbb{R}$, and we set $n_{0}:=n_{\mathcal{S}}+1, n_{1}:=n_{\mathcal{S}}$, and $n_{2}:=n_{\mathcal{S}}-1$. Moreover, from now on, if not stated otherwise, we always assume that $|\lambda|>0$. In order to apply the Sokhotski-Plemelj argument, we analyze the neighborhoods of the roots of $d_{0}$, located at

$$
k_{x}:=\frac{x \pi}{n_{0}},
$$

where $x \in M:=\left\{x \in \mathbb{Z}|| x \mid \leq n_{\mathcal{S}}+1\right\}$. The neighborhoods of these roots are denoted by $K_{x}:=\left(k_{x}-\kappa_{x}, k_{x}+\kappa_{x}\right) \cap(-\pi, \pi)$ and their size $\kappa_{x}$, satisfying $0<\kappa_{x} \leq k_{1} / 2$ for all $x \in M$, will be suitably chosen below. Moreover, for all $x \in M$, we define the integrals

$$
I_{\lambda, x}:=\lambda^{2} \int_{K_{x}} \frac{\mathrm{d} k}{2 \pi} \frac{N(k)}{D_{\lambda}(k)} .
$$

Then, we can make the decomposition

$$
\frac{2 J_{\lambda}}{\lambda^{2}}=\sum_{x \in M_{0}} I_{\lambda, x}+\sum_{x \in M \backslash M_{0}} I_{\lambda, x}+\lambda^{2} \int_{K^{c}} \frac{\mathrm{d} k}{2 \pi} \frac{N(k)}{D_{\lambda}(k)},
$$

where we set $M_{0}:=\left\{0, \pm n_{0}\right\}$ and $K^{c}:=(-\pi, \pi) \backslash\left(\cup_{x \in M} K_{x}\right)$. In the following, we will successively study all the contributions of the different integration domains in the decomposition (84). The coupling strength will always be assumed sufficiently small without 
necessarily specifying its size in each estimate. Moreover, if nothing else is indicated, the estimates are supposed to hold for all momenta. Finally, the positive constant $C$ can take different values at each place it appears.

Case 1: $K_{x}$ for $x \in M_{0}$ Let us set $\kappa_{x}:=\kappa_{0}:=k_{1} / 2$ for all $x \in M_{0}$ and let us rewrite (72) by using the identity from Remark 12. Then, after an eventual shift of (83) to the origin, we have $\left|N\left(k_{x}+k\right)\right| \leq C k^{2}$ and $d_{0}\left(k_{x}+k\right) \geq C k^{2}$ for $|k|<\kappa_{0}$. Since $\sum_{i=1}^{4}\left|d_{2 i}\left(k_{x}+k\right)\right| \leq C k^{2}$, we get $D_{\lambda}\left(k_{x}+k\right) \geq C k^{2}$ for $|k|<\kappa_{0}$. Hence, for $x \in M_{0}$ and $\lambda \rightarrow 0$, we find

$$
I_{\lambda, x}=\mathcal{O}\left(\lambda^{2}\right) .
$$

Case 2: $K_{x}$ for $x \in M \backslash M_{0}$

In order to determine some size $\kappa_{x}$ for the neighborhood $K_{x}$, we estimate (75) from below as follows. First, we define the function $d_{4^{\prime \prime}}: \mathbb{R} \rightarrow \mathbb{R}$ by $d_{4^{\prime \prime}}:=4 \sigma_{n_{1}}^{2} \sigma^{2}$ which allows us to write (75), shifted to the origin, in the form

$$
\begin{aligned}
R_{\lambda}\left(k_{x}+k\right) & =d_{4^{\prime \prime}}\left(k_{x}\right) \\
& +d_{4^{\prime \prime}}\left(k_{x}+k\right)-d_{4^{\prime \prime}}\left(k_{x}\right) \\
& +d_{4}\left(k_{x}+k\right)-d_{4^{\prime}}\left(k_{x}+k\right)-d_{4^{\prime \prime}}\left(k_{x}+k\right) \\
& +\lambda^{2} d_{6}\left(k_{x}+k\right) \\
& +\lambda^{4} d_{8}\left(k_{x}+k\right) .
\end{aligned}
$$

Using $|(87)|,|(88)| \leq C|k|$ and $|(89)|,|(90)| \leq C$, we have $R_{\lambda}\left(k_{x}+k\right) \geq 4 \sigma^{4}\left(k_{x}\right)-C\left(|k|+\lambda^{2}\right)$. Moreover, we note that $\sigma^{4}\left(k_{x}\right) \geq \sigma^{4}\left(k_{1}\right)=: \vartheta>0$ for all $x \in M \backslash M_{0}$. Hence, there exists a $\kappa>0$ which we can choose as $\kappa:=\vartheta /\left(3 n_{0}\right) \leq 1 / 6$, s.t., for $|k|<\kappa$, we have

$$
R_{\lambda}\left(k_{x}+k\right) \geq \vartheta
$$

Therefore, we set $\kappa_{x}:=\kappa$ for all $x \in M \backslash M_{0}$. In order to study (83) in the neighborhood $K_{x}=\left(k_{x}-\kappa, k_{x}+\kappa\right)$, we make the decomposition $I_{\lambda, x}=A_{\lambda, x}+N\left(k_{x}\right) B_{\lambda, x} / n_{0}$, where

$$
\begin{aligned}
A_{\lambda, x} & :=\lambda^{2} \int_{K_{x}} \frac{\mathrm{d} k}{2 \pi} \frac{N(k)-N\left(k_{x}\right)}{D_{\lambda}(k)}, \\
B_{\lambda, x} & :=n_{0} \lambda^{2} \int_{K_{x}} \frac{\mathrm{d} k}{2 \pi} \frac{1}{D_{\lambda}(k)} .
\end{aligned}
$$

Let us first analyze (92). To this end, we further decompose it as $A_{\lambda, x}=A_{\lambda, x}^{(1)}+A_{\lambda, x}^{(2)}$, where

$$
\begin{aligned}
& A_{\lambda, x}^{(1)}:=\lambda^{2} \int_{0}^{\kappa} \frac{\mathrm{d} k}{2 \pi} \frac{N_{x}^{+}(k) L_{\lambda}\left(k_{x}-k\right)+N_{x}^{-}(k) L_{\lambda}\left(k_{x}+k\right)}{D_{\lambda}\left(k_{x}+k\right) D_{\lambda}\left(k_{x}-k\right)}, \\
& A_{\lambda, x}^{(2)}:=\lambda^{6} \int_{0}^{\kappa} \frac{\mathrm{d} k}{2 \pi} \frac{N_{x}^{+}(k) R_{\lambda}\left(k_{x}-k\right)+N_{x}^{-}(k) R_{\lambda}\left(k_{x}+k\right)}{D_{\lambda}\left(k_{x}+k\right) D_{\lambda}\left(k_{x}-k\right)},
\end{aligned}
$$


and the functions $N_{x}^{ \pm}: \mathbb{R} \rightarrow \mathbb{R}$ are defined by $N_{x}^{ \pm}(k):=N\left(k_{x} \pm k\right)-N\left(k_{x}\right)$. Next, let us decompose (94) as $A_{\lambda, x}^{(1)}=A_{\lambda, x}^{(1,1)}+A_{\lambda, x}^{(1,2)}$, where

$$
\begin{aligned}
& A_{\lambda, x}^{(1,1)}:=\lambda^{2} \int_{0}^{\kappa} \frac{\mathrm{d} k}{2 \pi} \frac{N_{x}^{+}(k) d_{0}\left(k_{x}-k\right)+N_{x}^{-}(k) d_{0}\left(k_{x}+k\right)}{D_{\lambda}\left(k_{x}+k\right) D_{\lambda}\left(k_{x}-k\right)}, \\
& A_{\lambda, x}^{(1,2)}:=\lambda^{4} \int_{0}^{\kappa} \frac{\mathrm{d} k}{2 \pi} \frac{N_{x}^{+}(k)\left(d_{2}+\lambda^{2} d_{4^{\prime}}\right)\left(k_{x}-k\right)+N_{x}^{-}(k)\left(d_{2}+\lambda^{2} d_{4^{\prime}}\right)\left(k_{x}+k\right)}{D_{\lambda}\left(k_{x}+k\right) D_{\lambda}\left(k_{x}-k\right)} .
\end{aligned}
$$

In order to bound (96), we use $d_{0}\left(k_{x} \pm k\right)=d_{0}(k)$ for all $x \in M$, expand $N \in C^{\infty}(\mathbb{R} \backslash \pi \mathbb{Z})$ around $k_{x}$ up to second order in $\pm k$, and apply $D_{\lambda}\left(k_{x} \pm k\right) \geq L_{\lambda}\left(k_{x} \pm k\right)+\vartheta \lambda^{4}>0$ for all $|k|<\kappa$ which follows from (91) and the fact that $L_{\lambda}=\ell_{\lambda}^{2} \geq 0$, where $\ell_{\lambda}: \mathbb{R} \rightarrow \mathbb{R}$ is

$$
\ell_{\lambda}:=\sigma_{n_{0}}-2 \lambda^{2} \sigma_{n_{1}} \epsilon
$$

Hence, we get

$$
\left|A_{\lambda, x}^{(1,1)}\right| \leq C \lambda^{2} \int_{0}^{\kappa} \frac{\mathrm{d} k}{2 \pi} \frac{k^{2} d_{0}(k)}{\left(\left[\ell_{\lambda}\left(k_{x}+k\right)\right]^{2}+\vartheta \lambda^{4}\right)\left(\left[\ell_{\lambda}\left(k_{x}-k\right)\right]^{2}+\vartheta \lambda^{4}\right)} .
$$

Since $d_{0}=\sigma_{n_{0}}^{2}$ and $\ell_{\lambda}\left(k_{x} \pm k\right)=\sigma_{n_{0}}(k) \pm 2(-1)^{x+1} \lambda^{2} \sigma\left(n_{1}\left[k_{x} \pm k\right]\right) \epsilon\left(k_{x} \pm k\right)$, we make the coordinate transformation $k=\arcsin \left(\lambda^{2} p\right) / n_{0}$ for $0 \leq p \leq a_{\lambda}:=\sigma\left(n_{0} \kappa\right) / \lambda^{2}$, where $n_{0} \kappa<\pi / 2$. Hence, we get

$$
\mid \text { r.h.s. }(99) \mid \leq C \int_{0}^{a_{\lambda}} \frac{\mathrm{d} p}{2 \pi} \frac{p^{2}\left[\arcsin \left(\lambda^{2} p\right)\right]^{2}}{\left(\left[p+\ell_{\lambda, x}^{+}(p)\right]^{2}+\vartheta\right)\left(\left[p+\ell_{\lambda, x}^{-}(p)\right]^{2}+\vartheta\right)},
$$

where, for $\Lambda_{\lambda}:=\left(-1 / \lambda^{2}, 1 / \lambda^{2}\right)$, the functions $\ell_{\lambda, x}^{ \pm}: \Lambda_{\lambda} \rightarrow \mathbb{R}$ are defined by

$$
\begin{aligned}
\ell_{\lambda, x}^{ \pm}(p) & :=\left[\ell_{1}-\ell_{0}\right]\left(k_{x} \pm \arcsin \left(\lambda^{2} p\right) / n_{0}\right) \\
& = \pm 2(-1)^{x+1} \sigma\left(n_{1}\left[k_{x} \pm \arcsin \left(\lambda^{2} p\right) / n_{0}\right]\right) \epsilon\left(k_{x} \pm \arcsin \left(\lambda^{2} p\right) / n_{0}\right)
\end{aligned}
$$

Since $\left|\ell_{\lambda, x}^{ \pm}(p)\right| \leq 2$ for all $p \in \Lambda_{\lambda}$, we find

$$
\mid \text { r.h.s. }|100| \leq C \lambda^{4}\left(\int_{0}^{2} \frac{\mathrm{d} p}{2 \pi} \frac{p^{4}}{\vartheta^{2}}+\int_{2}^{a_{\lambda}} \frac{\mathrm{d} p}{2 \pi} \frac{p^{4}}{\left([p-2]^{2}+\vartheta\right)^{2}}\right) .
$$

The integrand of the second integral on the r.h.s. of (102) is bounded and, hence, due to $a_{\lambda}=\sigma(\vartheta / 3) / \lambda^{2}$, we find $A_{\lambda, x}^{(1,1)}=\mathcal{O}\left(\lambda^{2}\right)$. We next turn to the estimate of (97). For this purpose, we make the decomposition $A_{\lambda, x}^{(1,2)}=A_{\lambda, x}^{(1,2,1)}+A_{\lambda, x}^{(1,2,2)}$, where

$$
\begin{aligned}
& A_{\lambda, x}^{(1,2,1)}:=\lambda^{4} \int_{0}^{\kappa} \frac{\mathrm{d} k}{2 \pi} \frac{N_{x}^{+}(k) d_{2}\left(k_{x}-k\right)+N_{x}^{-}(k) d_{2}\left(k_{x}+k\right)}{D_{\lambda}\left(k_{x}+k\right) D_{\lambda}\left(k_{x}-k\right)}, \\
& A_{\lambda, x}^{(1,2,2)}:=\lambda^{6} \int_{0}^{\kappa} \frac{\mathrm{d} k}{2 \pi} \frac{N_{x}^{+}(k) d_{4^{\prime}}\left(k_{x}-k\right)+N_{x}^{-}(k) d_{4^{\prime}}\left(k_{x}+k\right)}{D_{\lambda}\left(k_{x}+k\right) D_{\lambda}\left(k_{x}-k\right)} .
\end{aligned}
$$


In order to bound (103), we first bound the numerator in (103) by using $\left|N_{x}^{ \pm}(k)\right| \leq C k$ and $\left|d_{2}\left(k_{x} \pm k\right)\right| \leq 4 \sigma\left(n_{0} k\right)$ for all $k \in[0, \kappa]$, and we treat the denominator in (103) as in (99). Then, proceeding as in (100), we can write

$$
\left|A_{x}^{(1,2,1)}(\lambda)\right| \leq C \int_{0}^{a_{\lambda}} \frac{\mathrm{d} p}{2 \pi} \frac{p \arcsin \left(\lambda^{2} p\right)}{\left(\left[p+\ell_{\lambda, x}^{+}(p)\right]^{2}+\vartheta\right)\left(\left[p+\ell_{\lambda, x}^{-}(p)\right]^{2}+\vartheta\right)} .
$$

Moreover, analogously to (102), we get

$$
\mid \text { r.h.s. } 105 \mid \leq C \lambda^{2}\left(\int_{0}^{2} \frac{\mathrm{d} p}{2 \pi} \frac{p^{2}}{\vartheta^{2}}+\int_{2}^{a_{\lambda}} \frac{\mathrm{d} p}{2 \pi} \frac{p^{2}}{\left([p-2]^{2}+\vartheta\right)^{2}}\right) \text {. }
$$

Extending the integration domain of the second integral on the r.h.s. of (106) to infinity, we get $A_{\lambda, x}^{(1,2,1)}=\mathcal{O}\left(\lambda^{2}\right)$. In order to bound (104), using $\left|d_{4^{\prime}}\left(k_{x} \pm k\right)\right| \leq C$ and again $\left|N_{x}^{ \pm}(k)\right| \leq C k$ for all $k \in[0, \kappa]$, we can proceed as above and get

$$
\left|A_{\lambda, x}^{(1,2,2)}\right| \leq C \lambda^{2}\left(\int_{0}^{2} \frac{\mathrm{d} p}{2 \pi} \frac{p}{\vartheta^{2}}+\int_{2}^{a_{\lambda}} \frac{\mathrm{d} p}{2 \pi} \frac{p}{\left([p-2]^{2}+\vartheta\right)^{2}}\right)
$$

which again implies that $A_{\lambda, x}^{(1,2,2)}=\mathcal{O}\left(\lambda^{2}\right)$. Finally, in order to bound (95), we note that $\left|R_{\lambda}\left(k_{x} \pm k\right)\right| \leq C$, and estimating (95) as (104), we get $A_{\lambda, x}^{(2)}=\mathcal{O}\left(\lambda^{2}\right)$. Taking all of the foregoing estimates together finally implies that the term (92) does not contribute anything to the second order of the current, i.e., for $\lambda \rightarrow 0$, we have $A_{\lambda, x}=\mathcal{O}\left(\lambda^{2}\right)$ for all $x \in M \backslash M_{0}$. We next turn to the study of (93). For this purpose, we rewrite (93) using the coordinate transformation introduced before (100) which leads to

$$
B_{\lambda, x}=\int_{-a_{\lambda}}^{a_{\lambda}} \frac{\mathrm{d} p}{2 \pi} Y_{\lambda, x}(p)
$$

where the function $Y_{\lambda, x}: \Lambda_{\lambda} \rightarrow \mathbb{R}$ is defined by

$$
Y_{\lambda, x}(p):=\frac{1}{\left(1-\lambda^{4} p^{2}\right)^{1 / 2}} \frac{1}{\left[p+\ell_{\lambda, x}(p)\right]^{2}+R_{\lambda, x}(p)},
$$

and we set $\ell_{\lambda, x}:=\ell_{\lambda, x}^{+}$, and the function $R_{\lambda, x}: \Lambda_{\lambda} \rightarrow \mathbb{R}$ is given by $R_{\lambda, x}(p):=R_{\lambda}\left(k_{x}+\right.$ $\left.\arcsin \left(\lambda^{2} p\right) / n_{0}\right)$. Let us first write $B_{\lambda, x}=B_{0, x}+\left[B_{\lambda, x}-B_{0, x}\right]$, where

$$
B_{0, x}:=\int_{-\infty}^{\infty} \frac{\mathrm{d} p}{2 \pi} Y_{0, x}(p)
$$

the function $Y_{0, x}: \mathbb{R} \rightarrow \mathbb{R}$ is defined by

$$
Y_{0, x}(p):=\frac{1}{\left[p+\ell_{0, x}\right]^{2}+R_{0, x}}
$$


and the constants are given, for all $x \in M \backslash M_{0}$, by $\ell_{0, x}:=\sigma_{2}\left(k_{x}\right)$ and $R_{0, x}:=4 \sigma^{4}\left(k_{x}\right)>0$. Let us next decompose the difference as $B_{\lambda, x}-B_{0, x}=B_{\lambda, x}^{(1)}-B_{\lambda, x}^{(2)}$, where

$$
\begin{aligned}
B_{\lambda, x}^{(1)} & :=\int_{-a_{\lambda}}^{a_{\lambda}} \frac{\mathrm{d} p}{2 \pi}\left[Y_{\lambda, x}(p)-Y_{0, x}(p)\right], \\
B_{\lambda, x}^{(2)} & :=\int_{|p| \geq a_{\lambda}} \frac{\mathrm{d} p}{2 \pi} Y_{0, x}(p) .
\end{aligned}
$$

Furthermore, we make the decomposition $B_{\lambda, x}^{(1)}=B_{\lambda, x}^{(1,1)}+B_{\lambda, x}^{(1,2)}$, where

$$
\begin{aligned}
B_{\lambda, x}^{(1,1)} & :=\lambda^{4} \int_{-a_{\lambda}}^{a_{\lambda}} \frac{\mathrm{d} p}{2 \pi} \frac{p^{2}}{\left(1-\lambda^{4} p^{2}\right)^{1 / 2}\left(1+\left(1-\lambda^{4} p^{2}\right)^{1 / 2}\right)} \frac{1}{\left[p+\ell_{\lambda, x}(p)\right]^{2}+R_{\lambda, x}(p)} \\
B_{\lambda, x}^{(1,2)} & :=\int_{-a_{\lambda}}^{a_{\lambda}} \frac{\mathrm{d} p}{2 \pi}\left(\frac{1}{\left[p+\ell_{\lambda, x}(p)\right]^{2}+R_{\lambda, x}(p)}-\frac{1}{\left[p+\ell_{0, x}\right]^{2}+R_{0, x}}\right) .
\end{aligned}
$$

In order to bound (114), we use $\left(1-\lambda^{4} p^{2}\right)^{1 / 2} \geq \epsilon(\vartheta / 3)$ for all $|p| \leq a_{\lambda},\left|\ell_{\lambda, x}(p)\right| \leq 2$, and (91) which yields

$$
\left|B_{\lambda, x}^{(1,1)}\right| \leq C \lambda^{4}\left(\int_{0}^{2} \frac{\mathrm{d} p}{2 \pi} \frac{p^{2}}{\vartheta}+\int_{2}^{a_{\lambda}} \frac{\mathrm{d} p}{2 \pi} \frac{p^{2}}{(p-2)^{2}+\vartheta}\right) .
$$

Due to the boundedness of the integrand of the second integral, we have $B_{\lambda, x}^{(1,1)}=\mathcal{O}\left(\lambda^{2}\right)$. In order to estimate (115), we make the decomposition $B_{\lambda, x}^{(1,2)}=B_{\lambda, x}^{(1,2,1)}+B_{\lambda, x}^{(1,2,2)}$, where

$$
\begin{aligned}
B_{\lambda, x}^{(1,2,1)} & :=\int_{-a_{\lambda}}^{a_{\lambda}} \frac{\mathrm{d} p}{2 \pi} \frac{\left[p+\ell_{0, x}\right]^{2}-\left[p+\ell_{\lambda, x}(p)\right]^{2}}{\left(\left[p+\ell_{0, x}\right]^{2}+R_{0, x}\right)\left(\left[p+\ell_{\lambda, x}(p)\right]^{2}+R_{\lambda, x}(p)\right)}, \\
B_{\lambda, x}^{(1,2,2)} & :=\int_{-a_{\lambda}}^{a_{\lambda}} \frac{\mathrm{d} p}{2 \pi} \frac{R_{0, x}-R_{\lambda, x}(p)}{\left(\left[p+\ell_{0, x}\right]^{2}+R_{0, x}\right)\left(\left[p+\ell_{\lambda, x}(p)\right]^{2}+R_{\lambda, x}(p)\right)} .
\end{aligned}
$$

In order to bound (117), we use $\left|\left[p+\ell_{0, x}\right]^{2}-\left[p+\ell_{\lambda, x}(p)\right]^{2}\right| \leq C(1+|p|)\left|\ell_{0, x}-\ell_{\lambda, x}(p)\right|$ and $\left|\ell_{0, x}-\ell_{\lambda, x}(p)\right| \leq C \lambda^{2}|p|$ for the numerator and, for the denominator, $\left|\ell_{0, x}\right|,\left|\ell_{\lambda, x}(p)\right| \leq 2$ and (91) which implies

$$
\left|B_{\lambda, x}^{(1,2,1)}\right| \leq C \lambda^{2}\left(\int_{0}^{2} \frac{\mathrm{d} p}{2 \pi} \frac{p}{\vartheta^{2}}+\int_{2}^{a_{\lambda}} \frac{\mathrm{d} p}{2 \pi} \frac{p^{2}}{\left([p-2]^{2}+\vartheta\right)^{2}}\right) .
$$

Extending the second integral to infinity, we get $B_{\lambda, x}^{(1,2,1)}=\mathcal{O}\left(\lambda^{2}\right)$. In order to bound (118), we use $\left|R_{0, x}-R_{\lambda, x}(p)\right| \leq C(1+|p|) \lambda^{2}$ and estimate the denominator as above yielding

$$
\left|B_{\lambda, x}^{(1,2,2)}\right| \leq C \lambda^{2}\left(\int_{0}^{2} \frac{\mathrm{d} p}{2 \pi} \frac{1}{\vartheta^{2}}+\int_{2}^{a_{\lambda}} \frac{\mathrm{d} p}{2 \pi} \frac{p}{\left([p-2]^{2}+\vartheta\right)^{2}}\right) .
$$


Extending the second integral to infinity, we again get $B_{\lambda, x}^{(1,2,2)}=\mathcal{O}\left(\lambda^{2}\right)$. We next turn to the estimate of (113). Again, from $\left|\ell_{0, x}\right| \leq 2$ and $R_{0, x} \geq \vartheta$, we have

$$
\left|B_{\lambda, x}^{(2)}\right| \leq C \int_{a_{\lambda}}^{\infty} \frac{\mathrm{d} p}{2 \pi} \frac{1}{[p-2]^{2}+\vartheta}
$$

and a coordinate transformation fixing the lower limit of the integral leads to $B_{\lambda, x}^{(2)}=\mathcal{O}\left(\lambda^{2}\right)$. Collecting the estimates for (92) and (93), we get that, for $x \in M \backslash M_{0}$ and $\lambda \rightarrow 0$,

$$
I_{\lambda, x}=\frac{N\left(k_{x}\right)}{n_{0}} \int_{-\infty}^{\infty} \frac{\mathrm{d} p}{2 \pi} Y_{0, x}(p)+\mathcal{O}\left(\lambda^{2}\right) .
$$

It remains to study the last term in (84).

Case 3: $K^{c}$

Rewriting the numerator of the integrand with the help of the identity from Remark 12 , we immediately get $|N(k)| \leq C$. Moreover, the zeroth order contribution of the denominator is bounded from below by $d_{0}(k) \geq d_{0}(\kappa)>0$ for all $k \in K^{c}$. Since we have $\left|D_{\lambda}(k)-d_{0}(k)\right| \leq$ $C \lambda^{2}$, we get $D_{\lambda}(k) \geq d_{0}(\kappa) / 2$ for all $k \in K^{c}$. Hence, for $\lambda \rightarrow 0$, we find

$$
\int_{K^{c}} \frac{\mathrm{d} k}{2 \pi} \frac{N(k)}{D_{\lambda}(k)}=\mathcal{O}(1)
$$

We can now extract the nontrivial second order contribution to the NESS current from (84). Using (85), 122), and 123), it follows from (84) that

$$
\begin{aligned}
\lim _{\lambda \rightarrow 0} \frac{J_{\lambda}}{\lambda^{2}} & =\frac{1}{2} \sum_{x \in M \backslash M_{0}} \frac{N\left(k_{x}\right)}{n_{0}} \int_{-\infty}^{\infty} \frac{\mathrm{d} p}{2 \pi} Y_{0, x}(p) \\
& =\frac{1}{4 n_{0}} \sum_{x \in M \backslash M_{0}} \frac{N\left(k_{x}\right)}{R_{0, x}^{1 / 2}} .
\end{aligned}
$$

This concludes the proof of the theorem.

\section{Van Hove regime}

We start this section by introducing what we call the product setting. In this setting, the sample algebra is split off from the total algebra, and we can conveniently focus on the thermodynamics of the sample system. It is defined as follows.

Definition 16 (Product setting) The ingredients for this setting, partially labelled by a tilde, are specified as follows. 
(a) Observable algebra

The observable algebras of the sample and the reservoir are defined by

$$
\begin{aligned}
\mathcal{O}_{\mathcal{S}} & :=\mathfrak{A}\left(\mathfrak{h}_{\mathcal{S}}\right), \\
\mathcal{O}_{\mathcal{R}} & :=\mathfrak{A}\left(\mathfrak{h}_{\mathcal{R}}\right),
\end{aligned}
$$

and the total observable algebra is defined to be their tensor product,

$$
\tilde{\mathcal{O}}:=\mathcal{O}_{\mathcal{S}} \otimes \mathcal{O}_{\mathcal{R}}
$$

(b) Dynamics

The Hamiltonians of the sample, the reservoir, the decoupled, and the coupled system are specified by

$$
\begin{aligned}
H_{\mathcal{S}} & :=\mathrm{d} \Gamma\left(h_{\mathcal{S}}\right), \\
H_{\mathcal{R}} & :=\mathrm{d} \Gamma\left(h_{\mathcal{R}}\right), \\
\tilde{H}_{0} & :=H_{\mathcal{S}} \otimes 1+1 \otimes H_{\mathcal{R}} \\
\tilde{H}_{\lambda, a} & :=\tilde{H}_{0}+\lambda \tilde{V}_{a} \\
\tilde{H}_{\lambda} & :=\tilde{H}_{0}+\lambda \tilde{V}
\end{aligned}
$$

where the couplings $\tilde{V}_{a}, \tilde{V} \in \tilde{\mathcal{O}}$ are given by

$$
\begin{aligned}
\tilde{V}_{a} & :=-1 \Gamma(-1) \otimes 1 \operatorname{Im}\left[a^{*}\left(i_{\mathcal{S}}^{*} \delta_{\mathcal{S}, a}\right) \otimes a\left(i_{\mathcal{R}}^{*} \delta_{\mathcal{R}, a}\right)\right], \\
\tilde{V} & :=\sum_{a \in\{L, R\}} \tilde{V}_{a} .
\end{aligned}
$$

Correspondingly, the dynamics $\tau_{\mathcal{S}}^{t} \in \operatorname{Aut}\left(\mathcal{O}_{\mathcal{S}}\right), \tau_{\mathcal{R}}^{t} \in \operatorname{Aut}\left(\mathcal{O}_{\mathcal{R}}\right)$, and $\tilde{\tau}_{\lambda, a}^{t}, \tilde{\tau}_{\lambda}^{t} \in \operatorname{Aut}(\tilde{\mathcal{O}})$ are given, for all $A$ in $\mathcal{O}_{\mathcal{S}}, \mathcal{O}_{\mathcal{R}}$, and $\tilde{\mathcal{O}}$, respectively, by

$$
\begin{aligned}
\tau_{\mathcal{S}}^{t}(A) & :=\mathrm{e}^{1 t H_{\mathcal{S}}} A \mathrm{e}^{-1 t H_{\mathcal{S}}}, \\
\tau_{\mathcal{R}}^{t}(A) & :=\mathrm{e}^{1 t H_{\mathcal{R}}} A \mathrm{e}^{-1 t H_{\mathcal{R}}}, \\
\tilde{\tau}_{\lambda, a}^{t}(A) & :=\mathrm{e}^{1 t \tilde{H}_{\lambda, a}} A \mathrm{e}^{-1 t \tilde{H}_{\lambda, a}}, \\
\tilde{\tau}_{\lambda}^{t}(A) & :=\mathrm{e}^{1 t \tilde{H}_{\lambda}} A \mathrm{e}^{-1 t \tilde{H}_{\lambda}} .
\end{aligned}
$$

(c) Initial state

The initial state of the sample $\omega_{\mathcal{S}} \in \mathcal{E}\left(\mathcal{O}_{\mathcal{S}}\right)$ and of the reservoir $\omega_{\mathcal{R}} \in \mathcal{E}\left(\mathcal{O}_{\mathcal{R}}\right)$ are defined to be quasifree with the densities $\rho_{\mathcal{S}} \in \mathcal{L}\left(\mathfrak{h}_{\mathcal{S}}\right)$ and $\rho_{\mathcal{R}} \in \mathcal{L}\left(\mathfrak{h}_{\mathcal{R}}\right)$, respectively. The total initial state $\tilde{\omega} \in \mathcal{E}(\tilde{\mathcal{O}})$ is defined by

$$
\tilde{\omega}:=\omega_{\mathcal{S}} \otimes \omega_{\mathcal{R}} .
$$


In order to show the equivalence of this product setting and the quasifree setting from Definition 1, we make use of the following lemma. We denote by $\mathcal{U}(\mathcal{H})$ the unitary operators on the Hilbert space $\mathcal{H}$.

Lemma 17 (Exponential law for fermions) For $i=1,2$, let $\mathfrak{F}\left(\mathfrak{h}_{i}\right)$ be the fermionic Fock spaces over the Hilbert spaces $\mathfrak{h}_{i}$ having vacua $\Omega_{i}$, creation and annihilation operators $a_{i}^{*}, a_{i}: \mathfrak{h}_{i} \rightarrow \mathcal{L}\left(\mathfrak{F}\left(\mathfrak{h}_{i}\right)\right)$, and second quantizations $\Gamma_{i}: \mathcal{U}\left(\mathfrak{h}_{i}\right) \rightarrow \mathcal{L}\left(\mathfrak{F}\left(\mathfrak{h}_{i}\right)\right)$. Then, there exists a unique $U \in \mathcal{U}\left(\mathfrak{F}\left(\mathfrak{h}_{1} \oplus \mathfrak{h}_{2}\right), \mathfrak{F}\left(\mathfrak{h}_{1}\right) \otimes \mathfrak{F}\left(\mathfrak{h}_{2}\right)\right)$ s.t.

$$
\begin{aligned}
U \Omega & =\Omega_{1} \otimes \Omega_{2}, \\
U a\left(f_{1} \oplus f_{2}\right) U^{*} & =a_{1}\left(f_{1}\right) \otimes 1_{2}+\Gamma_{1}\left(-1_{1}\right) \otimes a_{2}\left(f_{2}\right), \\
U \Gamma\left(U_{1} \oplus U_{2}\right) U^{*} & =\Gamma_{1}\left(U_{1}\right) \otimes \Gamma_{2}\left(U_{2}\right),
\end{aligned}
$$

where $\Omega, a^{*}$, a, and $\Gamma$ are the corresponding objects for $\mathfrak{F}\left(\mathfrak{h}_{1} \oplus \mathfrak{h}_{2}\right)$.

Proof. See, for example, Alicki and Fannes [1].

The two settings are then equivalent in the following sense.

Lemma 18 (Product setting isomorphism) Let $\Phi: \mathcal{L}\left(\mathfrak{F}\left(\mathfrak{h}_{\mathcal{S}} \oplus \mathfrak{h}_{\mathcal{R}}\right)\right) \rightarrow \mathcal{L}\left(\mathfrak{F}\left(\mathfrak{h}_{\mathcal{S}}\right) \otimes \mathfrak{F}\left(\mathfrak{h}_{\mathcal{R}}\right)\right)$ be defined by $\Phi(A):=U A U^{*}$, where $U \in \mathcal{U}\left(\mathfrak{F}\left(\mathfrak{h}_{\mathcal{S}} \oplus \mathfrak{h}_{\mathcal{R}}\right), \mathfrak{F}\left(\mathfrak{h}_{\mathcal{S}}\right) \otimes \mathfrak{F}\left(\mathfrak{h}_{\mathcal{R}}\right)\right)$ is the unitary from Lemma 17 corresponding to the decomposition $\mathfrak{h} \simeq \mathfrak{h}_{\mathcal{S}} \oplus \mathfrak{h}_{\mathcal{R}}$. Then, $\Phi$ is a $C^{*}$ algebra *-isomorphism. Moreover, the following assertions hold.

(a) $\Phi: \mathcal{O} \rightarrow \tilde{\mathcal{O}}$ is a $C^{*}$ algebra*-isomorphism.

(b) $\tilde{\tau}_{\lambda}^{t}=\Phi \circ \tau_{\lambda}^{t} \circ \Phi^{-1}$ for all $\lambda, t \in \mathbb{R}$

(c) $\tilde{\omega}=\omega \circ \Phi^{-1}$

Proof. Note that for our sample of finite size, $n_{\mathcal{S}}<\infty$, we have

$$
\Gamma(-1)=\prod_{x \in \mathbb{Z}_{\mathcal{S}}}\left(1-2 a^{*}\left(i_{\mathcal{S}}^{*} \delta_{x}\right) a\left(i_{\mathcal{S}}^{*} \delta_{x}\right)\right) \in \mathcal{O}_{\mathcal{S}}
$$

Moreover, the couplings are related by $\Phi\left(V_{\alpha}\right)=\tilde{V}_{\alpha}$. The proof is then analogous to the one of Aschbacher et al. [7], see there for details.

We next specify the van Hove weak coupling regime (see also Aschbacher et al. [7] for example). For this purpose, we make use of the weak coupling theory developed by Davies [13, 14] and summarized for our needs in Appendix C.

Definition 19 (Van Hove regime) Let the operator $P_{\mathcal{S}}: \tilde{\mathcal{O}} \rightarrow \mathcal{O}_{\mathcal{S}}$ be defined, for all $A \in$ $\mathcal{O}_{\mathcal{S}}$ and all $B \in \mathcal{O}_{\mathcal{R}}$, by

$$
P_{\mathcal{S}}(A \otimes B):=\omega_{\mathcal{R}}(B) A
$$


and the same notation is used for its extension to $\tilde{\mathcal{O}}$. Moreover, for $a \in\{L, R\}$, the twoparameter family of mappings $T_{\mathcal{S}, \lambda}^{t}, T_{\mathcal{S}, \lambda, a}^{t}: \mathcal{O}_{\mathcal{S}} \rightarrow \mathcal{O}_{\mathcal{S}}$ with $\lambda, t \in \mathbb{R}$ and $a \in\{L, R\}$ are defined, for all $A \in \mathcal{O}_{\mathcal{S}}$, by

$$
\begin{aligned}
T_{\mathcal{S}, \lambda}^{t}(A) & :=P_{\mathcal{S}}\left[\tilde{\tau}_{0}^{-t} \circ \tilde{\tau}_{\lambda}^{t}(A \otimes 1)\right], \\
T_{\mathcal{S}, \lambda, a}^{t}(A) & :=P_{\mathcal{S}}\left[\tilde{\tau}_{0}^{-t} \circ \tilde{\tau}_{\lambda, a}^{t}(A \otimes 1)\right] .
\end{aligned}
$$

The van Hove NESS $\omega_{\mathcal{S},+} \in \mathcal{E}\left(\mathcal{O}_{\mathcal{S}}\right)$ with density $\rho_{\mathcal{S},+} \in \mathcal{L}\left(\mathfrak{h}_{\mathcal{S}}\right)$ and the Davies generator $K_{H, a}: \mathcal{O}_{\mathcal{S}} \rightarrow \mathcal{O}_{\mathcal{S}}$ of subreservoir $a \in\{L, R\}$ are defined, for all $A \in \mathcal{O}_{\mathcal{S}}$, by

$$
\begin{aligned}
\omega_{\mathcal{S},+}(A) & :=\lim _{t \rightarrow \infty} \lim _{\lambda \rightarrow 0} \omega_{\mathcal{S}}\left(T_{\mathcal{S}, \lambda}^{t / \lambda^{2}}(A)\right), \\
K_{H, a}(A) & :=\left.\frac{\mathrm{d}}{\mathrm{d} t}\right|_{t=0} \lim _{\lambda \rightarrow 0} T_{\mathcal{S}, \lambda, a}^{t / \lambda^{2}}(A),
\end{aligned}
$$

if the limits exist. Finally, the van Hove energy current observable $\Phi_{\mathcal{S}, a} \in \mathcal{O}_{\mathcal{S}}$ and its expectation value $J_{\mathcal{S}, a}$ in the van Hove NESS are given by

$$
\begin{aligned}
\Phi_{\mathcal{S}, a} & :=K_{H, a}\left(H_{\mathcal{S}}\right), \\
J_{\mathcal{S}, a} & :=\omega_{\mathcal{S},+}\left(\Phi_{\mathcal{S}, a}\right) .
\end{aligned}
$$

Remark 20 For all $A \in \mathcal{O}_{\mathcal{S}}$ and $t>0$, defining

$$
K_{H}(A):=\left.\frac{\mathrm{d}}{\mathrm{d} t}\right|_{t=0} \lim _{\lambda \rightarrow 0} T_{\mathcal{S}, \lambda}^{t / \lambda^{2}}(A),
$$

and using $K_{H}=\sum_{a \in\{L, R\}} K_{H, a}$ (see, for example, Spohn and Lebowitz [22]), and the invariance of the van Hove NESS under the time evolution generated by $K_{H}$, we get the first law of thermodynamics of the van Hove regime,

$$
\sum_{a \in\{L, R\}} J_{\mathcal{S}, a}=0
$$

Hence, as for the microscopic regime, we set $J_{\mathcal{S}}:=J_{\mathcal{S}, L}$.

We begin our analysis by constructing the van Hove NESS. In the proof of the following theorem (and the subsequent one), we will make use of the reservoir time correlation function $\psi_{a}^{\beta}: \mathbb{R} \rightarrow \mathbb{C}$ with $a \in\{L, R\}$ and $\beta \in \mathbb{R}$ defined by

$$
\psi_{a}^{\beta}(t):=\left(\delta_{\mathcal{R}, a}, i_{a} \varrho_{\beta}\left(h_{a}\right) \mathrm{e}^{\mathrm{t} t h_{a}} i_{a}^{*} \delta_{\mathcal{R}, a}\right) .
$$

Moreover, we will use $\epsilon_{i}$ and $\pi_{i}$ with $i=1, \ldots, n_{\mathcal{S}}$ which are the simple eigenvalues and the corresponding eigenprojections of the sample Hamiltonian $h_{\mathcal{S}}$, respectively, given in Lemma 31 of Appendix A, and, for $a \in\{L, R\}$ and $i=1, \ldots, n_{\mathcal{S}}$, we set

$$
\Omega_{a, i}:=\left\|\pi_{i} i_{\mathcal{S}}^{*} \delta_{\mathcal{S}, a}\right\|^{2},
$$

and the scalar product and the norm in $\mathfrak{h}_{\mathcal{S}}$ are denoted as the ones in $\mathfrak{h}$. The NESS can then be characterized as follows. 
Theorem 21 (Van Hove NESS) There exists a unique quasifree van Hove NESS $\omega_{\mathcal{S},+} \in$ $\mathcal{E}\left(\mathcal{O}_{\mathcal{S}}\right)$ whose density has the form

$$
\rho_{\mathcal{S},+}=\frac{1}{2} \sum_{a \in\{L, R\}} \varrho_{\beta_{a}}\left(h_{\mathcal{S}}\right) .
$$

Proof. Let us introduce the two-parameter family of states $\omega_{\mathcal{S}, \lambda}^{t} \in \mathcal{E}\left(\mathcal{O}_{\mathcal{S}}\right)$ with $t, \lambda \in \mathbb{R}$ which, for all $A \in \mathcal{O}_{\mathcal{S}}$, is defined by

$$
\begin{aligned}
\omega_{\mathcal{S}, \lambda}^{t}(A) & :=\omega_{\mathcal{S}}\left(T_{\mathcal{S}, \lambda}^{t}(A)\right) \\
& =\tilde{\omega} \circ \tilde{\tau}_{\lambda}^{t}(A \otimes 1) .
\end{aligned}
$$

Lemma 18, (23), and (24) then imply that their two-point function can be written as

$$
\omega_{\mathcal{S}, \lambda}^{t}\left(a^{*}(f) a(g)\right)=\sum_{a \in\{\mathcal{S}, \mathcal{R}\}} F_{a}(\lambda, t)
$$

where, for fixed $f, g \in \mathfrak{h}_{\mathcal{S}}$, the function $F_{a}: \mathbb{R}^{2} \rightarrow \mathbb{C}$ with $a \in\{\mathcal{S}, \mathcal{R}\}$ is defined by

$$
F_{a}(\lambda, t):=\left(\mathrm{e}^{\mathrm{ith}} i_{\mathcal{S}} g, i_{a} \rho_{a} i_{a}^{*} \mathrm{e}^{\mathrm{i} t h_{\lambda}} i_{\mathcal{S}} f\right) .
$$

In order to study the limit for $\lambda \rightarrow 0$ of $F_{a}\left(\lambda, t / \lambda^{2}\right)$ with fixed $t>0$, we apply the weak coupling theory summarized in Appendix $\mathrm{C}$ in a form suitable for the present theorem (and for Theorem 22 below). Its ingredients are specified as follows: $\mathcal{H}:=\mathfrak{h}, P_{0}:=i_{\mathcal{S}} i_{\mathcal{S}}^{*}$, $P_{1}:=i_{\mathcal{R}} i_{\mathcal{R}}^{*}, U^{t}:=\mathrm{e}^{t Z}$ with $Z:=1 h_{0}$ (satisfying $\left[U^{t}, P_{0}\right]=0$ for all $t \in \mathbb{R}$ ), $A:=\sum_{a \in\{L, R\}} A_{a}$, $A_{a}:=1 v_{a}, V_{\lambda}^{t}:=\mathrm{e}^{\mathrm{ith}}, W_{\lambda}^{t}:=i_{\mathcal{S}} i_{\mathcal{S}}^{*} \mathrm{e}^{\mathrm{ith} h_{\lambda}} i_{\mathcal{S}} i_{\mathcal{S}}^{*}$ and $R_{\lambda}^{t}:=i_{\mathcal{R}} i_{\mathcal{R}}^{*} \mathrm{e}^{\mathrm{i} t h_{\lambda}} i_{\mathcal{S}} i_{\mathcal{S}}^{*}$. In order to simplify the verification of the assumptions of the weak coupling theory, we define the operator-valued function $A_{a, b, c}^{\beta}: \mathbb{R}^{3} \rightarrow \mathcal{L}(\mathfrak{h})$ with $a, b, c \in\{L, R\}$ and $\beta \in \mathbb{R}$ by

$$
\begin{aligned}
A_{a, b, c}^{\beta}(r, s, t) & :=2 U^{r} P_{0} A_{a} P_{1} B_{b}^{\beta} U^{s} P_{1} A_{c} P_{0} U^{t} \\
& =-\frac{1}{2} \delta_{a b} \delta_{a c} \psi_{a}^{\beta}(s)\left(\mathrm{e}^{-1 t h_{0}} \delta_{\mathcal{S}, a}, \cdot\right) \mathrm{e}^{1 r h_{0}} \delta_{\mathcal{S}, a},
\end{aligned}
$$

where we set $B_{b}^{\beta}:=i_{b} \varrho_{\beta}\left(h_{b}\right) i_{b}^{*}$ and the reservoir time correlation function $\psi_{a}^{\beta}$ is given in 153. Let us begin with the sample contribution. For $a=\mathcal{S}$, we can write (158) as

$$
F_{\mathcal{S}}(\lambda, t)=\frac{1}{2}\left(U^{-t} W_{\lambda}^{t} P_{0} i_{\mathcal{S}} g, U^{-t} W_{\lambda}^{t} P_{0} i_{\mathcal{S}} f\right) .
$$

In order to apply assertion (1) of Theorem 42 of Appendix C on each factor of the scalar product in (160), we verify the following three assumptions of Theorem 42. Assumption (a) is $\operatorname{dim}\left(\operatorname{ran}\left(P_{0}\right)\right)=n_{\mathcal{S}}<\infty$. Assumption (b) is $P_{0} A P_{0}=0$ and $P_{1} A P_{1}=0$ which follows from (15). It remains to verify assumption (c) which reads $\int_{0}^{\infty} \mathrm{d} t\left\|P_{0} A P_{1} U^{t} P_{1} A P_{0}\right\|<\infty$. Since $P_{1}=2 \sum_{b \in\{L, R\}} B_{b}^{0}$, we have

$$
\begin{aligned}
P_{0} A P_{1} U^{t} P_{1} A P_{0} & =\sum_{a, b, c \in\{L, R\}} A_{a, b, c}^{0}(0, t, 0) \\
& =-\frac{1}{2} \sum_{a \in\{L, R\}} \psi_{a}^{0}(t)\left(\delta_{\mathcal{S}, a} \cdot \cdot\right) \delta_{\mathcal{S}, a}
\end{aligned}
$$


from which it follows that $\left\|P_{0} A P_{1} U^{t} P_{1} A P_{0}\right\| \leq \frac{1}{2} \sum_{a \in\{L, R\}}\left|\psi_{a}^{0}(t)\right| \leq 1$. In order to analyze the temporal decay of (153), we proceed to the diagonalization of $h_{a}$ by using Lemma 29 of Appendix A. Switching to the energy space $\widetilde{\mathfrak{h}}_{+}=L^{2}\left([-1,1]\right.$; de) of $h_{a}$, we get, for all $\beta \in \mathbb{R}$ and $a \in\{L, R\}$, that

$$
\psi_{a}^{\beta}(t)=\frac{2}{\pi} \int_{-1}^{1} \mathrm{~d} e\left(1-e^{2}\right)^{1 / 2} \varrho_{\beta}(e) \mathrm{e}^{1 t e},
$$

which, by symmetry, is independent of $a$. From the asymptotic analysis of Lemma 43 of Appendix $\mathbf{C}$ (or by noting that, for the case $\beta=0$, we can write $\psi_{a}^{0}(t)=J_{1}(t) / t$, where $J_{1}$ is the first order Bessel function), we have, for $t \rightarrow \infty$, that

$$
\psi_{a}^{\beta}(t)=\mathcal{O}\left(t^{-3 / 2}\right) .
$$

Therefore, assumption (c) is also satisfied and we can apply assertion (1) of Theorem 42. This assertion implies that, for any fixed $t>0$, we get

$$
\lim _{\lambda \rightarrow 0} F_{\mathcal{S}}\left(\lambda, t / \lambda^{2}\right)=\left(g, \rho_{\mathcal{S} \mathcal{S}}^{t} f\right),
$$

where the operator $\rho_{\mathcal{S} \mathcal{S}}^{t} \in \mathcal{L}\left(\mathfrak{h}_{\mathcal{S}}\right)$ is defined, for all $t \in \mathbb{R}$, by

$$
\rho_{\mathcal{S S}}^{t}:=\frac{1}{2} i_{\mathcal{S}}^{*} \mathrm{e}^{t\left(K^{\natural}\right)} \mathrm{e}^{t K^{\natural}} i_{\mathcal{S}} .
$$

Here, $K^{\natural}=\sum_{a \in\{L, R\}} K_{a}^{\natural} \in \mathcal{L}(\mathfrak{h})$, where, for $a \in\{L, R\}$, the operator $K_{a}^{\natural} \in \mathcal{L}(\mathfrak{h})$ is the spectral average from Theorem 42 of the Davies generator $K_{a} \in \mathcal{L}(\mathfrak{h})$ given by

$$
\begin{aligned}
K_{a} & :=\int_{0}^{\infty} \mathrm{d} t U^{-t} P_{0} A_{a} P_{1} U^{t} P_{1} A_{a} P_{0} \\
& =\sum_{b \in\{L, R\}} \int_{0}^{\infty} \mathrm{d} t A_{a, b, a}^{0}(-t, t, 0) \\
& =-\frac{1}{2} \int_{0}^{\infty} \mathrm{d} t \psi_{a}^{0}(t)\left(\delta_{\mathcal{S}, a}, \cdot\right) \mathrm{e}^{-1 t h_{0}} \delta_{\mathcal{S}, a} .
\end{aligned}
$$

For the computation of $K_{a}^{\natural}$, we make use of the fact that, for any $A \in \mathcal{L}(\mathfrak{h})$, we can write $A^{\natural}=\sum_{i=1}^{n_{\mathcal{S}}} i_{\mathcal{S}} \pi_{i} i_{\mathcal{S}}^{*} A i_{\mathcal{S}} \pi_{i} i_{\mathcal{S}}^{*}$, where $h_{\mathcal{S}}=\sum_{i=1}^{n_{\mathcal{S}}} \epsilon_{i} \pi_{i}$ with $\pi_{i}:=\left(\varphi_{i}, \cdot\right) \varphi_{i}$ is the spectral representation of the sample Hamiltonian whose simple eigenvalues $\epsilon_{i}$ and the corresponding orthonormal eigenfunctions $\varphi_{i}$ are given in Lemma 31 of Appendix A. Using this representation, we find

$$
K_{a}^{\natural}=-\frac{1}{2} \sum_{i=1}^{n_{\mathcal{S}}} \Psi_{a}^{0}\left(1 \epsilon_{i}\right) \Omega_{a, i} i_{\mathcal{S}} \pi_{i} i_{\mathcal{S}}^{*},
$$

where $\Psi_{a}^{\beta}$ denotes the Laplace transform of $\psi_{a}^{\beta}$ and $\Omega_{a, i}$ is given in (154). Hence, for all $t \in \mathbb{R}$, we immediately get

$$
\mathrm{e}^{t K_{a}^{\natural}}=i_{\mathcal{R}} i_{\mathcal{R}}^{*}+\sum_{i=1}^{n_{\mathcal{S}}} \mathrm{e}^{-\frac{1}{2} t \Psi_{a}^{0}\left(1 \epsilon_{i}\right) \Omega_{a, i}} i_{\mathcal{S}} \pi_{i} i_{\mathcal{S}}^{*} .
$$


Using that $\left[K_{a}^{\natural}, K_{b}^{\natural}\right]=\left[K_{a}^{\natural},\left(K_{b}^{\natural}\right)^{*}\right]=0$ for all $a, b \in\{L, R\}$ and plugging (168) and its adjoint into (165), we find that, for any $t>0$, the sample contribution has the form

$$
\begin{aligned}
\rho_{\mathcal{S S}}^{t} & =\frac{1}{2} i_{\mathcal{S}}^{*} \prod_{a \in\{L, R\}} \mathrm{e}^{t\left(K_{a}^{\natural}\right)^{*}} \prod_{b \in\{L, R\}} \mathrm{e}^{t K_{b}^{\natural}} i_{\mathcal{S}} \\
& =\frac{1}{2} \sum_{i=1}^{n_{\mathcal{S}}} \prod_{a \in\{L, R\}} \mathrm{e}^{-t \operatorname{Re}\left[\Psi_{a}^{0}\left(1 \epsilon_{i}\right)\right] \Omega_{a, i}} \pi_{i} .
\end{aligned}
$$

We next turn to the reservoir contribution. For $a=\mathcal{R}$, we can write 158 as

$$
F_{\mathcal{R}}(\lambda, t)=\sum_{a \in\{L, R\}}\left(R_{\lambda}^{t} P_{0} i_{\mathcal{S}} g, B_{a}^{\beta_{a}} R_{\lambda}^{t} P_{0} i_{\mathcal{S}} f\right)
$$

and $B_{a}^{\beta_{a}} \geq 0$ and $\left[B_{a}^{\beta_{a}}, U^{t}\right]=0$ for all $t \in \mathbb{R}$ and all $a \in\{L, R\}$. In order to determine the limit for $\lambda \rightarrow 0$ of $F_{\mathcal{R}}\left(\lambda, t / \lambda^{2}\right)$ with fixed $t>0$, we apply assertion (2) of Theorem 42. To this end, we have to verify that $S:=\sum_{a, b, c \in\{L, R\}} S_{a, b, c}$ converges in norm, where

$$
\begin{aligned}
S_{a, b, c} & :=-2 \int_{0}^{\infty} \mathrm{d} t \operatorname{Re}\left[P_{0} A_{a} P_{1} B_{b}^{\beta_{b}} U^{t} P_{1} A_{c} P_{0} U^{-t}\right] \\
& =-\int_{0}^{\infty} \mathrm{d} t \operatorname{Re}\left[A_{a, b, c}^{\beta_{b}}(0, t,-t)\right] \\
& =\frac{1}{2} \delta_{a b} \delta_{a c} \int_{0}^{\infty} \mathrm{d} t \operatorname{Re}\left[\psi_{a}^{\beta_{a}}(t)\left(\mathrm{e}^{\mathrm{i} t h_{0}} \delta_{\mathcal{S}, a}, \cdot\right) \delta_{\mathcal{S}, a}\right] .
\end{aligned}
$$

Using again Lemma 43 , we get $\left\|\operatorname{Re}\left[P_{0} A_{a} P_{1} B_{b}^{\beta_{b}} U^{t} P_{1} A_{c} P_{0} U^{-t}\right]\right\| \leq \frac{1}{4}\left|\psi_{a}^{\beta_{a}}(t)\right|=\mathcal{O}\left(t^{-3 / 2}\right)$ for $t \rightarrow \infty$. Hence, we apply assertion (2) of Theorem 42 which implies, for any fixed $t>0$, that

$$
\lim _{\lambda \rightarrow 0} F_{\mathcal{R}}\left(\lambda, t / \lambda^{2}\right)=\left(g, \rho_{\mathcal{S R}}^{t} f\right),
$$

where $\rho_{\mathcal{S} \mathcal{R}}^{t} \in \mathcal{L}\left(\mathfrak{h}_{\mathcal{S}}\right)$ is defined, for all $t \in \mathbb{R}$, by

$$
\rho_{\mathcal{S R}}^{t}:=\int_{0}^{t} \mathrm{~d} s i_{\mathcal{S}}^{*} \mathrm{e}^{s\left(K^{\natural}\right)^{*}} S^{\natural} \mathrm{e}^{s K^{\natural}} i_{\mathcal{S}} .
$$

Using the spectral representation of the sample Hamiltonian $h_{\mathcal{S}}$ as above, we get

$$
S_{a, b, c}^{\natural}=\frac{1}{2} \delta_{a b} \delta_{a c} \sum_{i=1}^{n_{\mathcal{S}}} \operatorname{Re}\left[\Psi_{a}^{\beta_{a}}\left(1 \epsilon_{i}\right)\right] \Omega_{a, i} i_{\mathcal{S}} \pi_{i} i_{\mathcal{S}}^{*} .
$$

Plugging (168) and (174) into (173), we find that, for any $t>0$, the reservoir contributes as

$$
\begin{aligned}
\rho_{\mathcal{S R}}^{t} & =\frac{1}{2} \sum_{a \in\{L, R\}} \int_{0}^{t} \mathrm{~d} s i_{\mathcal{S}}^{*} \prod_{b \in\{L, R\}} \mathrm{e}^{s\left(K_{b}^{\natural}\right)^{*}} S_{a, a, a}^{\natural} \prod_{c \in\{L, R\}} \mathrm{e}^{s K_{c}^{\natural}} i_{\mathcal{S}} \\
& =\frac{1}{2} \sum_{i=1}^{n_{\mathcal{S}}} \frac{\sum_{a \in\{L, R\}} \operatorname{Re}\left[\Psi_{a}^{\beta_{a}}\left(1 \epsilon_{i}\right)\right] \Omega_{a, i}}{\sum_{b \in\{L, R\}} \operatorname{Re}\left[\Psi_{b}^{0}\left(1 \epsilon_{i}\right)\right] \Omega_{b, i}}\left[1-\prod_{c \in\{L, R\}} \mathrm{e}^{-t \operatorname{Re}\left[\Psi_{c}^{0}\left(1 \epsilon_{i}\right)\right] \Omega_{c, i}}\right] \pi_{i} .
\end{aligned}
$$


The denominator in 175 is strictly positive due to Lemma 43 and Lemma 31 which yield that, for $i=1, \ldots, n_{\mathcal{S}}$, we have

$$
\begin{aligned}
\operatorname{Re}\left[\Psi_{a}^{\beta}\left(1 \epsilon_{i}\right)\right] & =2\left(1-\epsilon_{i}^{2}\right)^{1 / 2} \varrho_{\beta}\left(\epsilon_{i}\right), \\
\Omega_{a, i} & =\frac{2}{n_{\mathcal{S}}+1}\left(1-\epsilon_{i}^{2}\right),
\end{aligned}
$$

where $\epsilon_{i}=\epsilon\left(k_{i}\right)$ and $k_{i}=i \pi /\left(n_{\mathcal{S}}+1\right)$, and both expressions are independent of $a \in\{L, R\}$. Using (169) and (175), we then find the density (155) since

$$
\begin{aligned}
\omega_{\mathcal{S},+}\left(a^{*}(f) a(g)\right) & =\lim _{t \rightarrow \infty} \lim _{\lambda \rightarrow 0} \omega_{\mathcal{S}, \lambda}^{t}\left(a^{*}(f) a(g)\right) \\
& =\lim _{t \rightarrow \infty}\left(g,\left[\rho_{\mathcal{S} \mathcal{S}}^{t}+\rho_{\mathcal{S} \mathcal{R}}^{t}\right] f\right) \\
& =\left(g, \rho_{\mathcal{S},+} f\right) .
\end{aligned}
$$

Moreover, it follows from the quasifreeness of the initial state and Lemma 18 that the van Hove NESS is again quasifree. This is the assertion.

Now we are able to determine the energy current expectation in the van Hove NESS or the van Hove entropy production given by (see, for example, Aschbacher et al. [7])

$$
\operatorname{Ep}_{\mathcal{S}}:=-\sum_{a \in\{L, R\}} \beta_{a} J_{\mathcal{S}, a}=2 \delta J_{\mathcal{S}}
$$

In the following, $\operatorname{tr}$ denotes the trace over $\mathfrak{h}_{\mathcal{S}}$.

Theorem 22 (Van Hove second law of thermodynamics) The van Hove entropy production has the form

$$
\operatorname{Ep}_{\mathcal{S}}=\frac{2 \delta}{n_{\mathcal{S}}+1} \operatorname{tr}\left[S_{0}\left(h_{\mathcal{S}}\right)\right]
$$

where $S_{0}$ is given in Theorem 13. Hence, if the system is truly out of equilibrium and $n>0$, the van Hove entropy production is strictly positive.

Proof. The van Hove energy current observable is given in Definition 19 by

$$
\begin{aligned}
\Phi_{\mathcal{S}, a} & =K_{H, a}\left(H_{\mathcal{S}}\right) \\
& =\left.\frac{\mathrm{d}}{\mathrm{d} t}\right|_{t=0} \lim _{\lambda \rightarrow 0} T_{\mathcal{S}, \lambda, a}^{t / \lambda^{2}}\left(H_{\mathcal{S}}\right) .
\end{aligned}
$$

Moreover, for $n \in \mathbb{N}$, we know from (128) that the sample Hamiltonian has the form

$$
H_{\mathcal{S}}=\frac{1}{2} \sum_{x \in \mathbb{Z}_{\mathcal{S}}^{\prime}}\left[a^{*}\left(i_{\mathcal{S}}^{*} \delta_{x}\right) a\left(i_{\mathcal{S}}^{*} \delta_{x+1}\right)+a^{*}\left(i_{\mathcal{S}}^{*} \delta_{x+1}\right) a\left(i_{\mathcal{S}}^{*} \delta_{x}\right)\right]
$$


where we set $\mathbb{Z}_{\mathcal{S}}^{\prime}:=\mathbb{Z}_{\mathcal{S}} \backslash\{n\}$. For $n=0$, we have $H_{\mathcal{S}}=0$ since $h_{\mathcal{S}}=0$. Let us first consider (146) on the observable $A=a^{*}(f) a(g)$ for any $f, g \in \mathfrak{h}_{\mathcal{S}}$. We then get

$$
T_{\mathcal{S}, \lambda, a}^{t}\left(a^{*}(f) a(g)\right)=\sum_{b \in\{\mathcal{S}, \mathcal{R}\}} G_{b, a}(\lambda, t)
$$

where, for fixed $f, g \in \mathfrak{h}_{\mathcal{S}}$, the map $G_{b, a}: \mathbb{R}^{2} \rightarrow \mathcal{O}_{\mathcal{S}}$ with $b \in\{\mathcal{S}, \mathcal{R}\}$ and $a \in\{L, R\}$ is defined by

$$
\begin{aligned}
G_{\mathcal{S}, a}(\lambda, t) & :=a^{*}\left(f_{\mathcal{S}, a}(\lambda, t)\right) a\left(g_{\mathcal{S}, a}(\lambda, t)\right) \\
G_{\mathcal{R}, a}(\lambda, t) & :=\omega_{\mathcal{R}}\left(a^{*}\left(f_{\mathcal{R}, a}(\lambda, t)\right) a\left(g_{\mathcal{R}, a}(\lambda, t)\right)\right) 1_{\mathcal{S}}
\end{aligned}
$$

and, for any $f \in \mathfrak{h}$, the function $f_{b, a}: \mathbb{R}^{2} \rightarrow \mathfrak{h}_{b}$ with $b \in\{\mathcal{S}, \mathcal{R}\}$ and $a \in\{L, R\}$ is given by

$$
f_{b, a}(\lambda, t):=i_{b}^{*} \mathrm{e}^{-1 t h_{0}} \mathrm{e}^{1 t h_{\lambda, a}} i_{\mathcal{S}} f .
$$

In order to study the limit $\lambda \rightarrow 0$ of $G_{b, a}\left(\lambda, t / \lambda^{2}\right)$ for fixed $t>0$, we again apply the weak coupling theory from Appendix $\mathrm{C}$ with similar ingredients as in the proof of Theorem

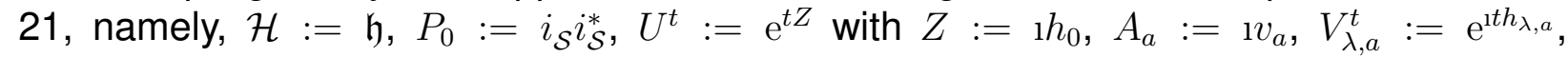
$W_{\lambda, a}^{t}:=i_{\mathcal{S}} i_{\mathcal{S}}^{*} \mathrm{e}^{\mathrm{ith} h_{\lambda, a}} i_{\mathcal{S}} i_{\mathcal{S}}^{*}$, and $R_{\lambda, a}^{t}:=i_{\mathcal{R}} i_{\mathcal{R}}^{*} \mathrm{e}^{1 t h_{\lambda, a}} i_{\mathcal{S}} i_{\mathcal{S}}^{*}$. Let us start with the sample contribution (184). Since $\|a(f)\|=\|f\|$ for all $f \in \mathfrak{h}_{\mathcal{S}}$, it is enough to study the weak coupling limit of

$$
f_{\mathcal{S}, a}(\lambda, t)=i_{\mathcal{S}}^{*} U^{-t} W_{\lambda, a}^{t} P_{0} i_{\mathcal{S}} f .
$$

The assumptions (a), (b), and (c) of Theorem 42 are again verified as in the proof of Theorem 21 with, in particular, $\left\|P_{0} A_{a} P_{1} U^{t} P_{1} A_{a} P_{0}\right\| \leq \frac{1}{2}\left|\psi_{a}^{0}(t)\right|=\mathcal{O}\left(t^{-3 / 2}\right)$ for $t \rightarrow \infty$, where here and in the following, we use the same notations as in the proof of Theorem 21. It then follows from assertion (1) of Theorem 42 and (168) that, for $t>0$, we have

$$
\begin{aligned}
\lim _{\lambda \rightarrow 0} f_{\mathcal{S}, a}\left(\lambda, t / \lambda^{2}\right) & =i_{\mathcal{S}}^{*} \mathrm{e}^{t K_{a}^{\natural}} P_{0} i_{\mathcal{S}} f \\
& =\sum_{i=1}^{n_{\mathcal{S}}} \mathrm{e}^{-\frac{1}{2} t \Psi_{a}^{0}\left(1 \epsilon_{i}\right) \Omega_{a, i}} \pi_{i} f .
\end{aligned}
$$

We next turn to the reservoir contribution (185). In this case, we have to study the weak coupling limit of

$$
G_{\mathcal{R}, a}(\lambda, t)=\sum_{b \in\{L, R\}}\left(R_{\lambda, a}^{t} P_{0} i_{\mathcal{S}} g, B_{b}^{\beta_{b}} R_{\lambda, a}^{t} P_{0} i_{\mathcal{S}} f\right) 1_{\mathcal{S}} .
$$

Since the additional assumption in assertion (2) of Theorem 42 about the norm convergence of (304) is satisfied due to (171) and the line following it, we get from (168) and (174) that, for $t>0$,

$$
\begin{aligned}
\lim _{\lambda \rightarrow 0} G_{\mathcal{R}, a}\left(\lambda, t / \lambda^{2}\right) & =\sum_{b \in\{L, R\}} \int_{0}^{t} \mathrm{~d} s\left(\mathrm{e}^{s K_{a}^{\natural}} P_{0} i_{\mathcal{S}} g, S_{a, b, a}^{\natural} \mathrm{e}^{s K_{a}^{\natural}} P_{0} i_{\mathcal{S}} f\right) 1_{\mathcal{S}} \\
& =\frac{1}{2} \sum_{i=1}^{n_{\mathcal{S}}} \frac{\operatorname{Re}\left[\Psi_{a}^{\beta_{a}}\left(1 \epsilon_{i}\right)\right]}{\operatorname{Re}\left[\Psi_{a}^{0}\left(1 \epsilon_{i}\right)\right]}\left[1-\mathrm{e}^{-t \operatorname{Re}\left[\Psi_{a}^{0}\left(1 \epsilon_{i}\right)\right] \Omega_{a, i}}\right]\left(g, \pi_{i} f\right) 1_{\mathcal{S}} .
\end{aligned}
$$


Therefore, from (188) and (190), we find

$$
\begin{aligned}
K_{H, a}\left(a^{*}(f) a(g)\right) & =\left.\sum_{b \in\{\mathcal{S}, \mathcal{R}\}} \frac{\mathrm{d}}{\mathrm{d} t}\right|_{t=0} \lim _{\lambda \rightarrow 0} G_{b, a}\left(\lambda, t / \lambda^{2}\right) \\
& =-\frac{1}{2} \sum_{i, j=1}^{n_{\mathcal{S}}}\left[\Psi_{a}^{0}\left(1 \epsilon_{i}\right) \Omega_{a, i}+\bar{\Psi}_{a}^{0}\left(1 \epsilon_{j}\right) \Omega_{a, j}\right] a^{*}\left(\pi_{i} f\right) a\left(\pi_{j} g\right) \\
& +\frac{1}{2} \sum_{i=1}^{n_{\mathcal{S}}} \operatorname{Re}\left[\Psi_{a}^{\beta_{a}}\left(1 \epsilon_{i}\right)\right] \Omega_{a, i}\left(g, \pi_{i} f\right) 1_{\mathcal{S}} .
\end{aligned}
$$

Applying (191) to (182), plugging the resulting expression into the van Hove NESS from Theorem 21, and using (176), 177), and $\sum_{x \in \mathbb{Z}_{\mathcal{S}}^{\prime}} \operatorname{Re}\left[\left(i_{\mathcal{S}}^{*} \delta_{x}, \pi_{i} i_{\mathcal{S}}^{*} \delta_{x+1}\right)\right]=\epsilon_{i}$, we arrive at the assertion.

We finally get the following result.

Theorem 23 (Van Hove is second order) The van Hove entropy production is the leading second order contribution to the small coupling expansion of the microscopic entropy production,

$$
\mathrm{Ep}_{\mathcal{S}}=\mathrm{Ep}
$$

Proof. Due to Lemma 31 in Appendix A which states that the eigenvalues of the sample Hamiltonian have the form $\epsilon_{i}=\epsilon\left(k_{i}\right)$ for all $i=1, \ldots, n_{\mathcal{S}}$, we immediately get the assertion by comparing (68) in Theorem 13 and (180) in Theorem 22.

Remark 24 In Aschbacher et al. [7], an assertion like Theorem 23 has been derived for the simple electronic black box model (SEBB) with one-dimensional sample system. The assumptions made there on the SEBB model compare to Lemma 30 and 31 of Appendix A. Definition 1, and Lemma 43 of Appendix C.

Remark 25 In Aschbacher and Spohn [9], a simple sufficient condition has been established which ensures the strict positivity of the entropy production as soon as the microscopic regime is related to the van Hove regime as in Theorem 23. In order to be able to apply this criterion, one assumption on the so-called effective coupling and another one on the triviality of some commutants have to be satisfied. Whereas it has been shown in [9] that the entropy production can still be strictly positive if the latter condition is violated, the present case is an example showing that the criterion is not necessary due to violation of the former condition. In order to formulate this condition precisely, we rewrite the couplings 1 as $\tilde{V}_{a}=\sum_{i=1}^{2} V_{\mathcal{S}, a, i}^{(1)} \otimes V_{\mathcal{R}, a, i}^{(1)}$, where $V_{\mathcal{S}, a, 1}^{(1)}=\operatorname{Re}\left[a\left(i_{\mathcal{S}}^{*} \delta_{\mathcal{S}, a}\right) \Gamma(-1)\right]$, 
$V_{\mathcal{S}, a, 2}^{(1)}=-\operatorname{Im}\left[a\left(i_{\mathcal{S}}^{*} \delta_{\mathcal{S}, a}\right) \Gamma(-1)\right]$, and

$$
\begin{aligned}
V_{\mathcal{R}, a, 1}^{(1)} & =\operatorname{Re}\left[a\left(i_{\mathcal{R}}^{*} \delta_{\mathcal{R}, a}\right)\right], \\
V_{\mathcal{R}, a, 2}^{(1)} & =\operatorname{Im}\left[a\left(i_{\mathcal{R}}^{*} \delta_{\mathcal{R}, a}\right)\right] .
\end{aligned}
$$

Moreover, we define the matrix-valued reservoir correlation function $R_{a}: \mathbb{R} \rightarrow \mathbb{C}^{2 \times 2}$ by

$$
R_{a, i j}(t):=\omega_{\mathcal{R}}\left(\tau_{\mathcal{R}}^{t}\left(V_{\mathcal{R}, a, i}^{(1)}\right) V_{\mathcal{R}, a, j}^{(1)}\right) .
$$

The effective coupling conditions from [9] then requires that, for all $a \in\{L, R\}$ and for all energies $\epsilon \in \operatorname{spec}\left(H_{\mathcal{S}}\right)-\operatorname{spec}\left(H_{\mathcal{S}}\right)$, the temporal Fourier transform of the reservoir correlation matrix should be positive definite,

$$
\hat{R}_{a}(\epsilon)>0 \text {. }
$$

Now, due to (128), and 207) from Appendix $\mathrm{A}$, we have on $\mathfrak{F}\left(\mathfrak{h}_{\mathcal{S}}\right)=\mathbb{C} \oplus\left(\oplus_{\alpha=1}^{n_{\mathcal{S}}} \mathfrak{h}_{\mathcal{S}}^{\wedge \alpha}\right)$ that

$$
\operatorname{spec}\left(H_{\mathcal{S}}\right)=\{0\} \cup\left(\bigcup_{\alpha=1}^{n_{\mathcal{S}}}\left\{\epsilon_{i_{1}}+\ldots+\epsilon_{i_{\alpha}}\right\}_{1 \leq i_{1}<\ldots<i_{\alpha} \leq n_{\mathcal{S}}}\right),
$$

where $\epsilon_{i}$ with $i=1, \ldots, n_{\mathcal{S}}$ are the eigenvalues of the one-particle sample Hamiltonian $h_{\mathcal{S}}$. On the other hand, due to the specific form of the Planck density, we have from (308) of Appendix $\mathrm{C}$ that $\hat{\psi}_{a}^{\beta}(-e)=\hat{\psi}_{a}^{-\beta}(e)=\mathrm{e}^{\beta e} \hat{\psi}_{a}^{\beta}(e)$ which, together with (195), yields

$$
\hat{R}_{a, i j}=\frac{\delta_{i j}}{2} \hat{\psi}_{a}^{\beta_{a}} \text {. }
$$

Choosing $n=1$, we have $\epsilon_{1}=-\epsilon_{3}=\sqrt{2} / 2$, and, hence, $\epsilon_{1}-\epsilon_{3}>1$. Since $\hat{\psi}_{a}^{\beta_{a}}\left(\epsilon_{1}-\epsilon_{3}\right)=0$ due to Lemma 43 in Appendix C, the effective coupling condition (196) is not satisfied for all energy differences.

Remark 26 As we indicated repeatedly in the appendix, the derivation of Theorem 23 for the full anisotropic $X Y$ model with an additional external magnetic field is much more complicated. This is also true for the derivation of a theorem like Theorem 23 for the isotropic case and general observables. We will study these question for more general quasifree systems elsewhere.

\section{A Spectral properties}

In this section, we display some spectral properties of the different Hamiltonians appearing in the model. In the first lemma, we introduce what we will call the energy space of the $X Y$ Hamiltonian $h$ being the direct integral decomposition of the absolutely continuous subspace w.r.t. which $h$ is diagonal, namely

$$
\widetilde{\mathfrak{h}}:=L^{2}\left([-1,1], \mathbb{C}^{2} ; \mathrm{d} e\right) .
$$


Moreover, the map $\tilde{\mathfrak{f}} \in \mathcal{L}(\widehat{\mathfrak{h}}, \widetilde{\mathfrak{h}})$ is defined, for all $\varphi \in \widehat{\mathfrak{h}}$, by

$$
\tilde{\mathfrak{f}} \varphi(e):=(2 \pi)^{-1 / 2}\left(1-e^{2}\right)^{-1 / 4}[\varphi(\arccos (e)), \varphi(-\arccos (e))],
$$

and the momentum space $\widehat{\mathfrak{h}}=L^{2}\left([-\pi, \pi] ; \frac{\mathrm{d} k}{2 \pi}\right)$ has been introduced in (32). We will use the notation $\tilde{f}:=\widetilde{\mathfrak{f} f} f$ for all $f \in \mathfrak{h}$, and $\widetilde{A}:=\widetilde{\mathfrak{f}} \mathfrak{f} A \mathfrak{f}^{*} \widetilde{\mathfrak{f}}^{*}$ for all $A \in \mathcal{L}(\mathfrak{h})$, where the Fourier transform $\mathfrak{f}: \mathfrak{h} \rightarrow \widehat{\mathfrak{h}}$ is also given after (32). Moreover, the Euclidean scalar product in the fiber $\mathbb{C}^{2}$ is denoted by $\langle\cdot, \cdot\rangle_{2}$.

Lemma 27 (XY Hamiltonian) The XY Hamiltonian $h \in \mathcal{L}(\mathfrak{h})$ has purely absolutely continuous spectrum with $\operatorname{spec}(h)=[-1,1]$, and it is diagonal in $\widetilde{\mathfrak{h}}$.

Proof. In momentum space $\widehat{\mathfrak{h}}$, the Hamiltonian $\widehat{h}$ acts as the multiplication by the dispersion relation $\epsilon(k)$ from Theorem 5. Moreover, a simple computation shows that $\widetilde{\mathfrak{f}}$ is a surjective isometry with $\widetilde{\mathfrak{f}}^{-1}=\widetilde{\mathfrak{f}}^{*}: \widetilde{\mathfrak{h}} \rightarrow \widehat{\mathfrak{h}}$ acting on all $\eta=:\left[\eta_{1}, \eta_{2}\right] \in \widetilde{\mathfrak{h}}$ as

$$
\tilde{\mathfrak{f}}^{*} \eta(k)=(2 \pi)^{1 / 2}\left(1-\epsilon^{2}(k)\right)^{1 / 4}\left[\chi_{[0, \pi]}(k) \eta_{1}(\epsilon(k))+\chi_{[-\pi, 0]}(k) \eta_{2}(\epsilon(k))\right] .
$$

This implies the assertion.

Remark 28 For $\gamma \neq 0$, the energy space for the $X Y$ Hamiltonian, now acting on $\mathfrak{h}^{\oplus 2}$ (see Remark 2), takes the form $L^{2}\left([-1,-|\gamma|], \mathbb{C}^{4} ; \mathrm{d} e\right) \oplus L^{2}\left([|\gamma|, 1], \mathbb{C}^{4} ; \mathrm{d} e\right)$ (and additional $\mathbb{C}^{2}$ valued factors if $\mu \neq 0$ ). Moreover, the nondiagonal matrix-multiplication operator by which its Fourier transform acts in momentum space $L^{2}\left([-\pi, \pi] ; \frac{\mathrm{d} k}{2 \pi}\right)^{\oplus 2}$ has to be diagonalized.

The subreservoir Hamiltonians have similar properties. Let us introduce the spaces $\mathfrak{h}_{+}:=\ell^{2}(\mathbb{N})$ and $\widehat{\mathfrak{h}}_{+}:=L^{2}\left([0, \pi] ; \frac{2}{\pi} \mathrm{d} k\right)$, and

$$
\widetilde{\mathfrak{h}}_{+}:=L^{2}([-1,1] ; \mathrm{d} e)
$$

Lemma 29 (Subreservoir Hamiltonians) The subreservoir Hamiltonians $h_{a} \in \mathcal{L}\left(\mathfrak{h}_{a}\right)$ with $a \in\{L, R\}$ have purely absolutely continuous spectrum with $\operatorname{spec}\left(h_{a}\right)=[-1,1]$, and they are diagonal in $\widetilde{\mathfrak{h}}_{+}$.

Proof. We use the unitary mappings $\mathfrak{h}_{a} \stackrel{t_{a}}{\rightarrow} \mathfrak{h}_{+} \stackrel{\mathfrak{s}}{\rightarrow} \widehat{\mathfrak{h}}_{+} \stackrel{\widetilde{\mathfrak{s}}}{\rightarrow} \widetilde{\mathfrak{h}}_{+}$, where the ingredients are given by $t_{L} f(x):=f(-(x+n))$ and $t_{R} f(x):=f(x+n)$ for all $f \in \mathfrak{h}_{a}$, by the Fourier-sine transform $\mathfrak{s}(f)(k):=\sum_{x=1}^{\infty} f(x) \sin (x k)$ for all $f \in \mathfrak{h}_{+}$, and by the energy transformation which, for all $\varphi \in \widehat{\mathfrak{h}}_{+}$, has the form

$$
\widetilde{\mathfrak{s}} \varphi(e):=2^{1 / 2} \pi^{-1 / 2}\left(1-e^{2}\right)^{-1 / 4} \varphi(\arccos (e)) .
$$


In $\widehat{\mathfrak{h}}_{+}$, the subreservoir Hamiltonians act by multiplication with $\epsilon(k)$, and applying the energy transformation, we get the assertion.

Next, we turn to the coupled and decoupled Hamiltonians. We denote by $\operatorname{spec}_{\mathrm{sc}}(A)$, $\operatorname{spec}_{\mathrm{ac}}(A)$, and $\operatorname{spec}_{\mathrm{pp}}(A)$ the singular continuous, the absolutely continuous, and the pure point spectrum of the operator $A$, respectively.

Lemma 30 ([De]coupled Hamiltonian) For all $\lambda \in \mathbb{R}$, it holds that $\operatorname{spec}_{\mathrm{sc}}\left(h_{\lambda}\right)=\emptyset$ and $\operatorname{spec}_{\mathrm{ac}}\left(h_{\lambda}\right)=[-1,1]$. Moreover, the coupled Hamiltonian has the properties $\operatorname{spec}_{\mathrm{pp}}\left(h_{\lambda}\right)=\emptyset$ for all $0<|\lambda| \leq 1$ and card $\left(\operatorname{spec}_{\mathrm{pp}}\left(h_{\lambda}\right)\right) \leq 2$ for all $|\lambda|>1$. The decoupled Hamiltonian satisfies $\operatorname{card}\left(\operatorname{spec}_{\mathrm{pp}}\left(h_{0}\right)\right)=n_{\mathcal{S}}$.

Proof. For the first two assertions and the fact that $\operatorname{card}\left(\operatorname{spec}_{\mathrm{pp}}\left(h_{\lambda}\right)\right)<\infty$ for all $\lambda \in \mathbb{R}$, see, for example, Hume and Robinson [16] (in fact, this is all what is used in Theorem 6). Next, let $\lambda \in \mathbb{R} \backslash\{0\}$, and let us assume that there exist $0 \neq f \in \mathfrak{h}$ and $e \in[-1,1]$ s.t. $h_{\lambda} f=e f$. Written out and evaluated at any $x \in \mathbb{Z}$, this equation reads

$$
f(x+1)+f(x-1)+(\lambda-1) \sum_{i=1}^{4}\left(\delta_{i}^{1}, f\right) \delta_{i}^{2}(x)=2 e f(x),
$$

where $\delta^{1}, \delta^{2} \in \mathfrak{h}^{4}$ are given in Lemma 32 of Appendix B. It follows from [16] that eigenfunctions corresponding to eigenvalues in $[-1,1]$ satisfy $f(x)=0$ for all $|x| \geq n+1$. Hence, plugging $x= \pm(n+1)$ into (204), we find that $f( \pm n)=0$. The eigenvalue equation then becomes $h_{\lambda} f=h f=$ ef which leads to $\operatorname{spec}_{\mathrm{pp}}\left(h_{\lambda}\right) \cap[-1,1]=\emptyset$ for all $|\lambda|>0$. Let us next consider the eigenvalue equation $h_{\lambda} f=e f$ for $0 \neq f \in \mathfrak{h}$ and $e \in \mathbb{R}$ with $|e|>1$ (see also Lemma 32 of Appendix B. Plugging $x= \pm n, \pm(n+1)$ into (204) and setting $f^{n}:=[f(-n), f(-n-1), f(n+1), f(n)] \in \mathbb{C}^{4}$, we get

$$
\Sigma_{\lambda}(e) f^{n}=0,
$$

where the matrix $\Sigma_{\lambda}(e) \in \mathbb{C}^{4 \times 4}$ is given in Lemma 32 and Lemma 36 of Appendix B. If $\operatorname{det}\left(\Sigma_{\lambda}(e)\right) \neq 0$, we again get $h_{\lambda} f=h f=e f$. Hence, the eigenvalues are the solutions of $\operatorname{det}\left(\Sigma_{\lambda}(e)\right)=0$, where, analogously to Proposition 38 of Appendix B, we have

$$
\operatorname{det}\left(\Sigma_{\lambda}\right)=\prod_{\sigma= \pm 1}\left[\left(1-\lambda^{2}\right) E^{2 n+2}+\sigma\left(\lambda^{2} E^{2}-1\right)\right],
$$

and $E(e)=e-\operatorname{sign}(e)\left(e^{2}-1\right)^{1 / 2}$ stems from (233) of Appendix B. Using that $0<E^{2}(e)<1$ for all $e \in \mathbb{R}$ with $|e|>1$, none of the two factors in (206) vanishes if $0<|\lambda| \leq 1$. On the other hand, if $|\lambda|>1$, the factor with $\sigma=1$ has at most one root (depending on the size of $|\lambda|$, it may have no root for small $n$ but for sufficiently large $n$, it has one root), whereas the factor with $\sigma=-1$ has exactly one root for all $n \in \mathbb{N}_{0}$. Finally, for $\lambda=0$, we know from Remark 3 , that $h_{0}$ does not couple the subsystems to each other. Using Lemma 29 and Lemma 31, we then arrive at the assertion. 
The spectral resolution of the sample Hamiltonian can be explicitly determined.

Lemma 31 (Sample Hamiltonian) The spectrum of the sample Hamiltonian $h_{\mathcal{S}} \in \mathcal{L}\left(\mathfrak{h}_{\mathcal{S}}\right)$ consists of $n_{\mathcal{S}}$ nondegenerate eigenvalues which, for $i=1, \ldots, n_{\mathcal{S}}$, have the form

$$
\epsilon_{i}=\epsilon\left(k_{i}\right), \quad k_{i}:=\frac{i \pi}{n_{\mathcal{S}}+1} .
$$

The corresponding orthonormal eigenfunctions $\varphi_{i} \in \mathfrak{h}_{\mathcal{S}}$ are given, for all $x \in \mathbb{Z}_{\mathcal{S}}$, by

$$
\varphi_{i}(x)=\left(\frac{2}{n_{\mathcal{S}}+1}\right)^{1 / 2} \sin \left(\left[x+\frac{n_{\mathcal{S}}+1}{2}\right] k_{i}\right) .
$$

Proof. Note that the sample Hamiltonian $h_{\mathcal{S}}=i_{\mathcal{S}}^{*} h i_{\mathcal{S}} \in \mathcal{L}\left(\mathfrak{h}_{\mathcal{S}} \simeq \mathbb{C}^{n_{\mathcal{S}}}\right)$ is the usual discrete Laplacian acting by application of the matrix $\left[h_{\mathcal{S}}\right]_{i j}=\frac{1}{2}\left(\delta_{i j+1}+\delta_{i j-1}\right)$ for $i, j=1, \ldots, n_{\mathcal{S}}$ (see, for example, Böttcher and Grudsky [10]).

\section{B Wave operator}

In this section, we use the stationary approach to scattering theory in order to compute the wave operators appearing in the NESS expectation value of the energy current observable. To this end, we first express the resolvent of the coupled Hamiltonian by the resolvent of the $\mathrm{XY}$ Hamiltonian. For any operator $A \in \mathcal{L}(\mathcal{H})$, we denote by $\operatorname{res}(A)$ the resolvent set of $A$ and by $r_{z}(A):=(A-z)^{-1} \in \mathcal{L}(\mathcal{H})$ the resolvent of $A$ at the point $z \in \operatorname{res}(A)$.

Lemma 32 (Coupled resolvent) For $z \in \operatorname{res}(h) \cap \operatorname{res}\left(h_{\lambda}\right)$, we have

$$
r_{z}\left(h_{\lambda}\right)=r_{z}(h)-\frac{\lambda-1}{2} \sum_{i, j=1}^{4} \Sigma_{\lambda, i j}^{-1}(z)\left(r_{\bar{z}}(h) \delta_{j}^{1}, \cdot\right) r_{z}(h) \delta_{i}^{2},
$$

where $v=\frac{1}{2} \sum_{i=1}^{4}\left(\delta_{i}^{1}, \cdot\right) \delta_{i}^{2}$ and $\delta^{1}:=\left[\delta_{i}^{1}\right]_{i=1}^{4}, \delta^{2}:=\left[\delta_{i}^{2}\right]_{i=1}^{4} \in \mathfrak{h}^{4}$ are given by

$$
\begin{aligned}
\delta^{1} & :=\left[\delta_{\mathcal{S}, L}, \delta_{\mathcal{R}, L}, \delta_{\mathcal{R}, R}, \delta_{\mathcal{S}, R}\right], \\
\delta^{2} & :=\left[\delta_{\mathcal{R}, L}, \delta_{\mathcal{S}, L}, \delta_{\mathcal{S}, R}, \delta_{\mathcal{R}, R}\right] .
\end{aligned}
$$

Moreover, the interaction matrix $\Sigma_{\lambda}(z) \in \mathbb{C}^{4 \times 4}$ is defined, for $i, j=1, \ldots, 4$, by

$$
\Sigma_{\lambda, i j}(z):=\delta_{i j}+\frac{\lambda-1}{2}\left(\delta_{i}^{1}, r_{z}(h) \delta_{j}^{2}\right)
$$


Remark 33 For $\gamma \neq 0$, the wave operator in the selfdual setting acts on $\mathfrak{h}^{\oplus 2}$ and has nonvanishing off-diagonal components (whereas for $\gamma=0$ it is block-diagonal). The interaction matrix then lies in $\mathbb{C}^{8 \times 8}$.

Proof. In order to simplify the notation, we drop the indices of the resolvents. Using the resolvent identity $r\left(h_{\lambda}\right)=r(h)-(\lambda-1) r(h) \operatorname{vr}\left(h_{\lambda}\right)$, we have, for all $f \in \mathfrak{h}$,

$$
r\left(h_{\lambda}\right) f+\frac{\lambda-1}{2} \sum_{j=1}^{4}\left(\delta_{j}^{1}, r\left(h_{\lambda}\right) f\right) r(h) \delta_{j}^{2}=r(h) f .
$$

Taking the scalar product of (213) with $\delta_{i}^{1}$ for all $i=1, \ldots, 4$, we get

$$
\left(1+\frac{\lambda-1}{2} A\right) \xi=\eta
$$

where the components of $\xi, \eta \in \mathbb{C}^{4}$ and $A \in \mathbb{C}^{4 \times 4}$ are defined, for $i, j=1, \ldots, 4$, by

$$
\begin{aligned}
\xi_{i} & :=\left(\delta_{i}^{1}, r\left(h_{\lambda}\right) f\right), \\
\eta_{i} & :=\left(\delta_{i}^{1}, r(h) f\right), \\
A_{i j} & :=\left(\delta_{i}^{1}, r(h) \delta_{j}^{2}\right) .
\end{aligned}
$$

Moreover, defining $B \in \mathbb{C}^{4 \times 4}$ by $B_{i j}:=\left(\delta_{i}^{1}, r\left(h_{\lambda}\right) \delta_{j}^{2}\right)$ for $i, j=1, \ldots, 4$, the resolvent identity implies that, for any $\lambda \in \mathbb{R}$, we have $\left(1+\frac{\lambda-1}{2} A\right)\left(1-\frac{\lambda-1}{2} B\right)=1$, so $1+\frac{\lambda-1}{2} A$ is invertible. We now solve (214) for $\xi$ and plug the resulting expression into (213). This yields the assertion.

We next introduce the following abbreviations.

Definition 34 (Boundary values) Let $z \in \operatorname{res}(h), e \in \mathbb{R}, \varepsilon>0$, and $f, g \in \mathfrak{h}$. We define

$$
\begin{aligned}
\varrho_{f, g}(z) & :=\left(f, r_{z}(h) g\right), \\
\gamma_{f, g}(e, \varepsilon) & :=\frac{1}{2 \pi 1}\left(\varrho_{f, g}(e+1 \varepsilon)-\varrho_{f, g}(e-1 \varepsilon)\right),
\end{aligned}
$$

and, if the limits exist, we write

$$
\begin{aligned}
\varrho_{f, g}(e \pm 10) & :=\lim _{\varepsilon \rightarrow 0^{+}} \varrho_{f, g}(e \pm 1 \varepsilon), \\
\gamma_{f, g}(e) & :=\lim _{\varepsilon \rightarrow 0^{+}} \gamma_{f, g}(e, \varepsilon) .
\end{aligned}
$$

Let us recall from Lemma 27 of Appendix $\mathrm{A}$ that $\widetilde{\mathfrak{h}}=L^{2}\left([-1,1], \mathbb{C}^{2} ; \mathrm{d} e\right)$ is the energy space of the $X Y$ Hamiltonian $h$. The wave operator then looks as follows.

Proposition 35 (Wave operator) In the energy space $\widetilde{\mathfrak{h}}$ of the $X Y$ Hamiltonian, the action of the wave operator is given, for all $f \in \mathfrak{h}$, by

$$
\widetilde{w}\left(h, h_{\lambda}\right) \widetilde{f}=\widetilde{f}-\frac{\lambda-1}{2} \sum_{i, j=1}^{4} \Sigma_{\lambda, i j}^{-1}(\cdot-10) \varrho_{\delta_{j}^{1}, f}(\cdot-10) \widetilde{\delta}_{i}^{2},
$$


where, for all $e \in(-1,1)$, the boundary interaction matrix $\Sigma_{\lambda}(e-10) \in \mathbb{C}^{4 \times 4}$ is defined by

$$
\Sigma_{\lambda, i j}(e-10):=\delta_{i j}+\frac{\lambda-1}{2} \varrho_{\delta_{i}^{1}, \delta_{j}^{2}}(e-10) .
$$

Proof. In order to compute the wave operator with the help of stationary scattering theory, we rewrite it in its weak abelian form (see, for example, Yafaev [23]),

$$
w\left(h, h_{\lambda}\right)=\underset{\varepsilon \rightarrow 0^{+}}{\mathrm{w}}-\lim _{0} 2 \varepsilon \int_{0}^{\infty} \mathrm{d} t \mathrm{e}^{-2 \varepsilon t} 1_{\mathrm{ac}}(h) \mathrm{e}^{-\mathrm{i} t h} \mathrm{e}^{\mathrm{i} t h_{\lambda}} 1_{\mathrm{ac}}\left(h_{\lambda}\right) .
$$

Applying Parseval's identity to (224), and using that $r_{e-1 \varepsilon}(h)=-1 \int_{0}^{\infty} \mathrm{d} t \mathrm{e}^{1 t(h-(e-1 \varepsilon))}$, we get, for all $f, g \in \mathfrak{h}$, that

$$
\left(f, w\left(h, h_{\lambda}\right) g\right)=\lim _{\varepsilon \rightarrow 0^{+}} \frac{\varepsilon}{\pi} \int_{-\infty}^{\infty} \mathrm{d} e\left(r_{e-1 \varepsilon}(h) 1_{\mathrm{ac}}(h) f, r_{e-1 \varepsilon}\left(h_{\lambda}\right) 1_{\mathrm{ac}}\left(h_{\lambda}\right) g\right) .
$$

Moreover, if the limit $\varepsilon \rightarrow 0^{+}$of $\varepsilon\left(r_{e-1 \varepsilon}(h) f, r_{e-1 \varepsilon}\left(h_{\lambda}\right) g\right)$ exists for all $f, g \in \mathfrak{h}$ and almost all $e \in \mathbb{R}$ (the set of full measure depending on $f$ and $g$ ) and using that $1_{\mathrm{ac}}(h)=1$ and $\operatorname{spec}(h)=[-1,1]$, we can write

$$
\left(f, w\left(h, h_{\lambda}\right) g\right)=\int_{-1}^{1} \mathrm{~d} e \lim _{\varepsilon \rightarrow 0^{+}} \frac{\varepsilon}{\pi}\left(r_{\mathrm{e}-1 \varepsilon}(h) f, r_{e-1 \varepsilon}\left(h_{\lambda}\right) g\right) .
$$

In order to compute the limit in (226), we express the resolvent $r_{e-1 \varepsilon}\left(h_{\lambda}\right)$ of the coupled Hamiltonian in terms of the resolvent $r_{e-1 \varepsilon}(h)$ of the XY Hamiltonian. Plugging (209) into the scalar product on the r.h.s. of (226), we have

$$
\frac{\varepsilon}{\pi}\left(r_{e-1 \varepsilon}(h) f, r_{e-1 \varepsilon}\left(h_{\lambda}\right) g\right)=\gamma_{f, g}(e, \varepsilon)-\frac{\lambda-1}{2} \sum_{i, j=1}^{4} \gamma_{f, \delta_{i}^{2}}(e, \varepsilon) \Sigma_{\lambda, i j}^{-1}(e-1 \varepsilon) \varrho_{\delta_{j}^{1}, g}(e-1 \varepsilon),
$$

where we used that $\frac{\varepsilon}{\pi}\left(r_{e-1 \varepsilon}(h) f, r_{e-1 \varepsilon}(h) g\right)=\gamma_{f, g}(e, \varepsilon)$ (which follows from the resolvent identity). Now, we know that, for all $f, g \in \mathfrak{h}$ and almost all $e \in[-1,1]$, the limit

$$
\varrho_{f, g}(e \pm 10)= \pm \pi \mathrm{l} \frac{\mathrm{d}(f, \zeta(e) g)}{\mathrm{d} e}+\mathrm{p} \cdot \mathrm{v} \cdot \int_{-1}^{1} \mathrm{~d} e^{\prime} \frac{1}{e^{\prime}-e} \frac{\mathrm{d}\left(f, \zeta\left(e^{\prime}\right) g\right)}{\mathrm{d} e^{\prime}}
$$

exists, where the p.v.-integral is Cauchy's principle value, the mapping $\zeta: \mathcal{B}(\mathbb{R}) \rightarrow \mathcal{L}(\mathfrak{h})$ denotes the projection-valued spectral measure of the $\mathrm{XY}$ Hamiltonian $h$ with $\mathcal{B}(\mathbb{R})$ the Borel sets on $\mathbb{R}$, and we used that

$$
\mathrm{d}(f, \zeta(e) g)=\chi_{[-1,1]}(e) \frac{\mathrm{d}(f, \zeta(e) g)}{\mathrm{d} e} \mathrm{~d} e .
$$

Moreover, it follows from (219) and 228 that

$$
\gamma_{f, g}(e)=\frac{\mathrm{d}(f, \zeta(e) g)}{\mathrm{d} e} .
$$


Hence, we find that

$$
\left(f, w\left(h, h_{\lambda}\right) g\right)=(f, g)-\frac{\lambda-1}{2} \sum_{i, j=1}^{4} \int_{-1}^{1} \mathrm{~d} e \gamma_{f, \delta_{i}^{2}}(e) \Sigma_{\lambda, i j}^{-1}(e-10) \varrho_{\delta_{j}^{1}, g}(e-10),
$$

where the invertibility of the interaction matrix is assured as in the proof of Lemma 32 and we used $\int_{-1}^{1} \mathrm{~d} e \gamma_{f, g}(e)=\left(f, 1_{\mathrm{ac}}(h) g\right)=(f, g)$ in the first term on the r.h.s. of (231). In order to write the derivatives in (230) entering (231) more explicitly, we switch to the energy space representation using $\mathfrak{h}$ of Lemma 27. This lemma implies that

$$
\frac{\mathrm{d}(f, \rho(e) g)}{\mathrm{d} e}=\langle\widetilde{f}(e), \widetilde{g}(e)\rangle_{2},
$$

where we recall that $\langle\cdot, \cdot\rangle_{2}$ denotes the Euclidean scalar product in the fiber $\mathbb{C}^{2}$ of the direct integral $\widetilde{\mathfrak{h}}$, and $\widetilde{f}=\widetilde{\mathfrak{f} f} f$ for all $f \in \mathfrak{h}$. Hence, plugging (230) and (232) into (231), we arrive at the assertion.

In order to completely determine the wave operator, we have to compute the boundary values and the inverse of the interaction matrix. To this end, we define the function $E: \mathbb{R} \rightarrow \mathbb{C}$ by

$$
E(e):= \begin{cases}e+1\left(1-e^{2}\right)^{1 / 2}, & \text { if }|e| \leq 1 \\ e-\operatorname{sign}(e)\left(e^{2}-1\right)^{1 / 2}, & \text { if }|e|>1 .\end{cases}
$$

Let us start with the computation of some $X Y$ resolvent amplitudes for completely localized wave functions (which is also used in Appendix A).

Lemma 36 (Resolvent amplitudes) For $x \in \mathbb{Z}$, we have

$$
-\frac{2 E^{|x|+1}(e)}{1-E^{2}(e)}= \begin{cases}\varrho_{\delta_{0}, \delta_{x}}(e-10), & \text { if }|e|<1 \\ \varrho_{\delta_{0}, \delta_{x}}(e), & \text { if }|e|>1\end{cases}
$$

Proof. Let $x \in \mathbb{Z}$ with $x \geq 0, e \in(-1,1)$, and $\varepsilon>0$ sufficiently small. We first rewrite the momentum space representation of the resolvent amplitude in the form of a contour integral over the positively oriented unit circle $\mathbb{T}$ as

$$
\varrho_{\delta_{0}, \delta_{x}}(e-1 \varepsilon)=\frac{1}{1 \pi} \oint_{\mathbb{T}} \mathrm{d} z \frac{z^{x}}{z^{2}-2(e-1 \varepsilon) z+1} .
$$

Then, using Cauchy's residue theorem and taking the limit $\epsilon \rightarrow 0^{+}$, we get the first expression in (234) for $x \geq 0$. Moreover, due to the parity invariance of the XY Hamiltonian, $[h, \theta]=0$, where $\theta: \mathfrak{h} \rightarrow \mathfrak{h}$ is defined, for all $f \in \mathfrak{h}$, by $(\theta f)(x):=f(-x)$, we also have $\varrho_{\delta_{0}, \delta_{-x}}(e-1 \epsilon)=\varrho_{\delta_{0}, \delta_{x}}(e-1 \epsilon)$. The second assertion is derived similarly. 
Remark 37 For $\gamma \neq 0$ and $\mu=0$, one gets an analogous expression for (235) but, in this case, there is a nontrivial numerator, and the polynomial in the denominator becomes biquadratic, $z^{4}+a z^{2}+1$, where $a$ depends on $\gamma, e$, and $\varepsilon$. Moreover, if both $\gamma \neq 0$ and $\mu \neq 0$, this polynomial changes to $z^{4}+a z^{3}+b z^{2}+a z+1$, where $a$ depends on $\mu$ and $\gamma$, and $b$ on $\mu, \gamma, e$, and $\varepsilon$. Hence, the computation of the roots becomes increasingly and substantially more complicated (see also, for example, Carey and Hume [11]).

We next turn to the computation of the inverse of the boundary value interaction matrix from Proposition 35. For the convenience of the reader who wants to work with this nonequilibrium model and for reasons of a possible future extension, we display the detailed results of the computations.

Proposition 38 (Inverse boundary interaction matrix) For all $e \in(-1,1)$, we have

$$
\Sigma_{\lambda}^{-1}(e-10)=\frac{1}{\Delta_{\lambda}(e)}\left[\begin{array}{cc}
M_{\lambda}(e) & N_{\lambda}(e) \\
\sigma_{1} N_{\lambda}(e) \sigma_{1} & \sigma_{1} M_{\lambda}(e) \sigma_{1}
\end{array}\right] .
$$

Here, the determinant $\Delta_{\lambda}:=\operatorname{det}\left[\Sigma_{\lambda}(\cdot-10)\right]:(-1,1) \rightarrow \mathbb{C}$ reads

$$
\Delta_{\lambda}=\frac{1-E^{4 n+4}}{\left(1-E^{2}\right)^{2}}-\frac{2 E^{2}\left(1-E^{4 n+2}\right)}{\left(1-E^{2}\right)^{2}} \lambda^{2}+\frac{E^{4}\left(1-E^{4 n}\right)}{\left(1-E^{2}\right)^{2}} \lambda^{4},
$$

the matrix-valued functions $M_{\lambda}, N_{\lambda}:(-1,1) \rightarrow \mathbb{C}^{2 \times 2}$ have the structure

$$
M_{\lambda}:=\left[\begin{array}{ll}
a_{\lambda} & b_{\lambda} \\
c_{\lambda} & a_{\lambda}
\end{array}\right], \quad N_{\lambda}:=\left[\begin{array}{ll}
d_{\lambda} & e_{\lambda} \\
e_{\lambda} & f_{\lambda}
\end{array}\right],
$$

and the functions $a_{\lambda}, \ldots, f_{\lambda}:(-1,1) \rightarrow \mathbb{C}$ are defined by

$$
\begin{aligned}
& a_{\lambda}:=\frac{1-E^{4 n+4}}{\left(1-E^{2}\right)^{2}}-\frac{E^{2}\left(1-E^{4 n+2}\right)}{\left(1-E^{2}\right)^{2}}\left(\lambda+\lambda^{2}\right)+\frac{E^{4}\left(1-E^{4 n}\right)}{\left(1-E^{2}\right)^{2}} \lambda^{3}, \\
& b_{\lambda}:=-\frac{E\left(1-E^{4 n+2}\right)}{\left(1-E^{2}\right)^{2}}(1-\lambda)+\frac{E^{3}\left(1-E^{4 n}\right)}{\left(1-E^{2}\right)^{2}}\left(\lambda^{2}-\lambda^{3}\right), \\
& c_{\lambda}:=-\frac{E\left(1-E^{4 n+4}\right)}{\left(1-E^{2}\right)^{2}}(1-\lambda)+\frac{E^{3}\left(1-E^{4 n+2}\right)}{\left(1-E^{2}\right)^{2}}\left(\lambda^{2}-\lambda^{3}\right), \\
& d_{\lambda}:=-\frac{E^{2 n+1}}{1-E^{2}}(1-\lambda), \\
& e_{\lambda}:=-\frac{E^{2 n+2}}{1-E^{2}}\left(\lambda-\lambda^{2}\right), \\
& f_{\lambda}:=-\frac{E^{2 n+3}}{1-E^{2}}\left(\lambda^{2}-\lambda^{3}\right) .
\end{aligned}
$$

Proof. For all $e \in(-1,1)$, the matrix $\Sigma_{\lambda}(e-10) \in \mathbb{C}^{4 \times 4}$ has the structure

$$
\Sigma_{\lambda}(e-10)=\left[\begin{array}{ll}
A_{\lambda}(e) & B_{\lambda}(e) \\
C_{\lambda}(e) & D_{\lambda}(e)
\end{array}\right]
$$


where the matrix-valued functions $A_{\lambda}, \ldots, D_{\lambda}:(-1,1) \rightarrow \mathbb{C}^{2 \times 2}$ are defined by

$$
\begin{aligned}
A_{\lambda} & :=\frac{1}{1-E^{2}}\left[\begin{array}{cc}
1-\lambda E^{2} & (1-\lambda) E \\
(1-\lambda) E & 1-\lambda E^{2}
\end{array}\right], \\
B_{\lambda} & :=(1-\lambda) \frac{E^{2 n+1}}{1-E^{2}}\left[\begin{array}{cc}
1 & E \\
E & E^{2}
\end{array}\right] \\
C_{\lambda} & :=\sigma_{1} B_{\lambda} \sigma_{1}, \\
D_{\lambda}: & :=A_{\lambda} .
\end{aligned}
$$

A lengthy calculation then leads to the assertion.

In the following lemmas, we display some ingredients used in the proof of Theorem 11. Recall from there that, for all $x \in \mathbb{Z}$ and $i, j=1, \ldots, 4$, the vector-valued functions $\xi_{x}, \eta_{x}:(-1,1) \rightarrow \mathbb{C}^{4}$ and the matrix-valued function $\Theta:(-1,1) \rightarrow \mathbb{C}^{4 \times 4}$ are given by

$$
\begin{aligned}
\xi_{x, i} & =\left\langle\widetilde{\delta}_{i}^{2}, \widetilde{\rho}_{1,+} \widetilde{\delta}_{x}\right\rangle_{2}, \\
\eta_{x, i} & =\varrho_{\delta_{i}^{1}, \delta_{x}}\left(\cdot-{ }_{10} 0,\right. \\
\Theta_{i j} & =\left\langle\widetilde{\delta}_{i}^{2}, \widetilde{\rho}_{1,+} \widetilde{\delta}_{j}^{2}\right\rangle_{2} .
\end{aligned}
$$

The first lemma displays the explicit form of these functions.

Lemma 39 (Ingredients, 1) For $x \in \mathbb{Z}$, the functions $\xi_{x}, \eta_{x}:(-1,1) \rightarrow \mathbb{C}^{4}$ and $\Theta$ : $(-1,1) \rightarrow \mathbb{C}^{4 \times 4}$ read

$$
\begin{aligned}
\xi_{x} & =\frac{E}{\pi 1\left(1-E^{2}\right)} \sum_{\sigma= \pm} \rho_{\sigma}\left[E^{\sigma(x+n+1)}, E^{\sigma(x+n)}, E^{\sigma(x-n)}, E^{\sigma(x-n-1)}\right], \\
\eta_{x} & =-\frac{2 E}{1-E^{2}}\left[E^{|x+n|}, E^{|x+n+1|}, E^{|x-n-1|}, E^{|x-n|}\right], \\
\Theta & =\frac{E}{\pi 1\left(1-E^{2}\right)} \sum_{\sigma= \pm} \rho_{\sigma}\left[\begin{array}{cccc}
1 & E^{\sigma} & E^{\sigma(2 n+1)} & E^{\sigma(2 n+2)} \\
E^{-\sigma} & 1 & E^{\sigma 2 n} & E^{\sigma(2 n+1)} \\
E^{-\sigma(2 n+1)} & E^{-\sigma 2 n} & 1 & E^{\sigma} \\
E^{-\sigma(2 n+2)} & E^{-\sigma(2 n+1)} & E^{-\sigma} & 1
\end{array}\right],
\end{aligned}
$$

where, for $\sigma= \pm$, the function $\varrho_{\sigma}: \mathbb{R} \rightarrow \mathbb{R}$ is defined by

$$
\varrho_{\sigma}(e):=\left(1+\mathrm{e}^{(\beta+\sigma \delta) e}\right)^{-1} .
$$

Moreover, for all $e \in(-1,1)$, we have

$$
\Sigma_{\lambda}^{-1}(e-10) \eta_{x}=\frac{1}{\Delta_{\lambda}(e)}\left[a_{\lambda, x}(e), b_{\lambda, x}(e), c_{\lambda, x}(e), d_{\lambda, x}(e)\right]
$$


where the component functions $a_{\lambda, x}, \ldots, d_{\lambda, x}:(-1,1) \rightarrow \mathbb{C}$ are given, for $x=n, 1, b y$

$$
\begin{aligned}
& a_{\lambda, n}:=-\frac{2 E^{2 n+1}}{1-E^{2}}, \\
& b_{\lambda, n}:=-\frac{2}{E^{2 n+2}} 1-E^{2} \lambda, \\
& c_{\lambda, n}:=-\frac{2 E^{2}\left(1-E^{4 n+2}\right)}{\left(1-E^{2}\right)^{2}} \lambda+\frac{2 E^{4}\left(1-E^{4 n}\right)}{\left(1-E^{2}\right)^{2}} \lambda^{3}, \\
& d_{\lambda, n}:=-\frac{2 E\left(1-E^{4 n+2}\right)}{\left(1-E^{2}\right)^{2}}+\frac{2 E^{3}\left(1-E^{4 n}\right)}{\left(1-E^{2}\right)^{2}} \lambda^{2},
\end{aligned}
$$

and, for $x=n+2$, by

$$
\begin{aligned}
a_{\lambda, n+2} & :=-\frac{2 E^{2 n+3}}{1-E^{2}} \lambda \\
b_{\lambda, n+2} & :=-\frac{2 E^{2 n+4}}{1-E^{2}} \lambda^{2}, \\
c_{\lambda, n+2} & :=-\frac{2 E^{2}\left(1-E^{4 n+4}\right)}{\left(1-E^{2}\right)^{2}}+\frac{2 E^{4}\left(1-E^{4 n+2}\right)}{\left(1-E^{2}\right)^{2}} \lambda^{2}, \\
d_{\lambda, n+2}: & :=-\frac{2 E^{3}\left(1-E^{4 n+2}\right)}{\left(1-E^{2}\right)^{2}} \lambda+\frac{2 E^{5}\left(1-E^{4 n}\right)}{\left(1-E^{2}\right)^{2}} \lambda^{3} .
\end{aligned}
$$

Proof. Note that, for all $x \in \mathbb{Z}$, we have

$$
\widetilde{\delta}_{x}=\left(\frac{E}{\pi_{1}\left(1-E^{2}\right)}\right)^{1 / 2}\left[E^{x}, E^{-x}\right],
$$

and that the density $\rho_{1,+} \in \mathcal{L}(\mathfrak{h})$ of the XY NESS given in Theorem 5 acts, for all $\eta \in \widetilde{\mathfrak{h}}$, as the matrix multiplication operator

$$
\widetilde{\rho}_{1,+} \eta=\operatorname{diag}\left(\varrho_{+}, \varrho_{-}\right) \eta .
$$

Hence, we get (253) and (255), and the expressions in (254) are given in Lemma 36 . Moreover, (257) directly follows from Proposition 38.

We next display some further ingredients used in the proof of Theorem 11. Recall from there that, for $x=n$ and $y=n+2$, the function $F_{\lambda}(x, y)=\left(w\left(h, h_{\lambda}\right) \delta_{x}, \rho_{1,+} w\left(h, h_{\lambda}\right) \delta_{y}\right)$ reads

$$
F_{\lambda}(n, n+2)=F_{1}(n, n+2)+\int_{-1}^{1} \mathrm{~d} e \frac{\sum_{i=0}^{8} p_{i}(e) \lambda^{i}}{\sum_{i=0}^{8} q_{i}(e) \lambda^{i}},
$$

where the coefficient functions $p_{i}, q_{i}:(-1,1) \rightarrow \mathbb{C}$ with $i=1, \ldots, 8$ are displayed in the following lemma.

\footnotetext{
${ }^{1}$ Due to their complicated form, we do not display the formulas for general $x \in \mathbb{Z}$.
} 
Lemma 40 (Ingredients, 2) The numerator functions $p_{i}:(-1,1) \rightarrow \mathbb{C}$ with $i=1, \ldots, 8$ have the form

$$
\begin{aligned}
& p_{0}=\frac{\left(1-E^{4 n+4}\right)^{2}}{\pi_{1} E^{4 n+1}\left(1-E^{2}\right)^{5}}\left(\varrho_{+} E^{4}+\varrho_{-}\right), \\
& p_{1}=\frac{\left(1+E^{2}\right)\left(1-E^{4 n+2}\right)\left(1-E^{4 n+4}\right)}{\pi_{1} E^{4 n+1}\left(1-E^{2}\right)^{3}} \varrho_{-}, \\
& p_{2}=-\frac{2\left(1+E^{2}\right)\left(1-E^{4 n+2}\right)\left(1-E^{4 n+4}\right)}{\pi_{1} E^{4 n+1}\left(1-E^{2}\right)^{5}}\left(\varrho_{+} E^{4}+\varrho_{-}\right), \\
& p_{3}=-\frac{\left(1+E^{8 n+4}\right)\left(1+3 E^{2}+E^{4}\right)-E^{4 n}\left(1+E^{2}+5 E^{4}+3 E^{6}\right)}{\pi_{1} E^{4 n+1}\left(1-E^{2}\right)^{3}} \varrho_{-} \\
& +\frac{E^{3}}{\pi_{1}\left(1-E^{2}\right)} \varrho_{+}, \\
& p_{4}=\frac{\left(1+E^{8 n+4}\right)\left(1+4 E^{2}+E^{4}\right)-E^{4 n}\left(1+10 E^{4}+E^{8}\right)}{\pi_{1} E^{4 n+1}\left(1-E^{2}\right)^{5}}\left(\varrho_{+} E^{4}+\varrho_{-}\right), \\
& p_{5}=\frac{2 E\left(1+E^{2}\right)\left(1-E^{4 n}\right)\left(1-E^{4 n+2}\right)}{\pi_{1} E^{4 n}\left(1-E^{2}\right)^{3}} \varrho_{-}, \\
& p_{6}=-\frac{2 E\left(1+E^{2}\right)\left(1-E^{4 n}\right)\left(1-E^{4 n+2}\right)}{\pi_{1} E^{4 n}\left(1-E^{2}\right)^{5}}\left(\varrho_{+} E^{4}+\varrho_{-}\right), \\
& p_{7}=-\frac{E^{3}\left(1-E^{4 n}\right)^{2}}{\pi_{1} E^{4 n}\left(1-E^{2}\right)^{3}} \varrho_{-}, \\
& p_{8}=\frac{E^{3}\left(1-E^{4 n}\right)^{2}}{\pi_{1} E^{4 n}\left(1-E^{2}\right)^{5}}\left(\varrho_{+} E^{4}+\varrho_{-}\right),
\end{aligned}
$$

and the denominator functions $q_{i}:(-1,1) \rightarrow \mathbb{C}$ with $i=1, \ldots, 8$ read $q_{1}=q_{3}=q_{5}=q_{7}=0$,

$$
\begin{aligned}
& q_{0}=-\frac{\left(1-E^{4 n+4}\right)^{2}}{E^{4 n}\left(1-E^{2}\right)^{4}} \\
& q_{2}=\frac{2\left(1+E^{2}\right)\left(1-E^{4 n+2}\right)\left(1-E^{4 n+4}\right)}{E^{4 n}\left(1-E^{2}\right)^{4}}, \\
& q_{4}=-\frac{\left(1+E^{4}\right)\left(1-E^{4 n}\right)\left(1-E^{4 n+4}\right)+4 E^{2}\left(1-E^{4 n+2}\right)^{2}}{E^{4 n}\left(1-E^{2}\right)^{4}}, \\
& q_{6}=\frac{2 E^{2}\left(1+E^{2}\right)\left(1-E^{4 n}\right)\left(1-E^{4 n+2}\right)}{E^{4 n}\left(1-E^{2}\right)^{4}} \\
& q_{8}=-\frac{E^{4}\left(1-E^{4 n}\right)^{2}}{E^{4 n}\left(1-E^{2}\right)^{4}} .
\end{aligned}
$$

Moreover, on the energies $e=\epsilon(k)$ with $k \in[0, \pi]$, we have $\operatorname{Im}\left[p_{2 i+1}(\epsilon(k))\right]=0$ for $i=0,2,3$ and, for $i=1$, we find

$$
\operatorname{Im}\left[p_{3}(\epsilon(k))\right]=-\frac{\epsilon(k)}{\pi}\left[\varrho_{\beta_{L}}(\epsilon(k))-\varrho_{\beta_{R}}(\epsilon(k))\right] .
$$


Moreover, on these energies, the denominator functions have the form

$$
\begin{gathered}
q_{0}(\epsilon(k))=\frac{\sin ^{2}(2(n+1) k)}{4 \sin ^{4}(k)}, \\
q_{2}(\epsilon(k))=-\frac{\sin ((2 n+1) k) \sin (2(n+1) k) \cos (k)}{\sin ^{4}(k)}, \\
q_{4}(\epsilon(k))=\frac{\sin (2 n k) \sin (2(n+1) k) \cos (2 k)+2 \sin ^{2}((2 n+1) k)}{2 \sin ^{4}(k)}, \\
q_{6}(\epsilon(k))=-\frac{\sin ((2 n+1) k) \sin (2 n k) \cos (k)}{\sin ^{4}(k)} \\
q_{8}(\epsilon(k))=\frac{\sin ^{2}(2 n k)}{4 \sin ^{4}(k)} .
\end{gathered}
$$

Proof. This follows from Lemma 39 and a lengthy calculation.

Finally, in the following lemma, we discuss the ingredients needed in order to derive the absolute convergence of the NESS current integral in the proof of Theorem 11. Recall from there that the function $Q_{\lambda}:(-1,1) \rightarrow \mathbb{R}$ is given by

$$
Q_{\lambda}=\left|\Delta_{\lambda}\right|^{2}=\mid \operatorname{det}\left(\left.\Sigma_{\lambda}(\cdot-10)\right|^{2}=\sum_{i=0}^{4} q_{2 i} \lambda^{2 i} .\right.
$$

This function has the following property.

Lemma 41 (Boundedness) For $\lambda \in \mathbb{R} \backslash\{0\}$, the inverse of $Q_{\lambda}$ is bounded. In particular, for $\lambda= \pm 1$, we have $Q_{ \pm 1}=1$.

Proof. For $\lambda=1$, we have $\Sigma_{1}(e-10)=1 \in \mathbb{C}^{4 \times 4}$ for all $e \in[-1,1]$ from (223). Since the determinant (237) contains even powers of $\lambda$ only, we thus have $Q_{ \pm 1}=1$ on $[-1,1]$. We next observe that

$$
\frac{1}{Q_{\lambda}}=-\frac{E^{4 n}\left(1-E^{2}\right)^{4}}{\prod_{i=1}^{4} P_{\lambda, i}}
$$

where the polynomials $P_{\lambda, i}:(-1,1) \rightarrow \mathbb{C}$ with $i=1, \ldots, 4$ are defined by

$$
\begin{aligned}
& P_{\lambda, 1}:=1+E^{2 n+2}-\left(1+E^{2 n}\right) \lambda^{2}, \\
& P_{\lambda, 2}:=-1+E^{2 n+2}+\left(1-E^{2 n}\right) \lambda^{2}, \\
& P_{\lambda, 3}:=1-E^{2 n+2}-E^{2}\left(1-E^{2 n}\right) \lambda^{2}, \\
& P_{\lambda, 4}:=-1-E^{2 n+2}+E^{2}\left(1+E^{2 n}\right) \lambda^{2} .
\end{aligned}
$$


From now on, let $\lambda \in \mathbb{R} \backslash\{0, \pm 1\}$. Then, it easily follows from (291)-(294) that, for all $n \in \mathbb{N}_{0}$, there are no unimodular roots of $P_{\lambda, 1}$ and $P_{\lambda, 4}$, whereas the only unimodular roots of $P_{\lambda, 2}$ and $P_{\lambda, 3}$ are $E= \pm 1$. We next study the order of these roots. Specializing (291) -294 for $n=0$, we see that, in this case, the roots are simple and we get

$$
\frac{1}{Q_{\lambda}}=-\frac{\left(1-E^{2}\right)^{2}}{\left(E^{2}+1-2 \lambda^{2}\right)\left(1+\left(1-2 \lambda^{2}\right) E^{2}\right)},
$$

which implies the assertion for $n=0$. Next, let $n>0$ and let us first consider $P_{\lambda, 2}$ and $E=1$. From the factorization $P_{\lambda, 2}=(E-1) R_{\lambda, 2}$, where we set

$$
R_{\lambda, 2}:=E^{2 n}(1+E)+\left(1-\lambda^{2}\right) \sum_{i=0}^{2 n-1} E^{i},
$$

we get $R_{\lambda, 2}(1)=2\left(n+1-n \lambda^{2}\right)$. Hence, if $\lambda^{2} \neq(n+1) / n$, the polynomial $P_{\lambda, 2}$ has a simple root at $E=1$. On the other hand, if $\lambda^{2}=(n+1) / n$, we can write $P_{\lambda, 2}=(E-1)^{2} S_{2}$, where

$$
S_{2}:=E^{2 n}+\frac{1}{n} \sum_{i=0}^{2 n-1}(2 n-i) E^{2 n-1-i},
$$

and now we have $S_{2}(1)=2(n+1) \neq 0$. Hence, in this case, $P_{\lambda, 2}$ has a double root at $E=1$. Since $P_{\lambda, 2}(-E)=P_{\lambda, 2}(E)$, the same conclusions hold for the root $E=-1$. Moreover, $P_{\lambda, 3}$ can be treated similarly (we again have the two cases $\lambda^{2}$ different or equal to $(n+1) / n)$ and the conclusions remain unchanged. Hence, the order of the roots $E= \pm 1$ in the denominator of 290 is not exceeding 4 and, since the numerator cancels these singularities of $1 / Q_{\lambda}$, we arrive at the assertion.

\section{Van Hove weak coupling theory}

The material of the following theorem is taken from Davies [13, 14]. We do not display his assertions in full generality but rather adapt them to our special case at hand. The ingredients are as follows. Let $\mathcal{H}$ be a Hilbert space, $P_{0} \in \mathcal{L}(\mathcal{H})$ a projection, and $P_{1}:=$ $1-P_{0} \in \mathcal{L}(\mathcal{H})$. Let $U^{t} \in \mathcal{L}(\mathcal{H})$ with $t \in \mathbb{R}$ be a strongly continuous one-parameter group of isometries s.t., for all $t \in \mathbb{R}$, we have

$$
\left[U^{t}, P_{0}\right]=0 .
$$

The generator of $U^{t}$ is denoted by $Z$. Moreover, let $A \in \mathcal{L}(\mathcal{H})$ with $A^{*}=-A$, and let $V_{\lambda}^{t} \in \mathcal{L}(\mathcal{H})$ with $\lambda, t \in \mathbb{R}$ be the one-parameter group generated by $Z+\lambda A$. Besides, the operators $W_{\lambda}^{t}, R_{\lambda}^{t} \in \mathcal{L}(\mathcal{H})$ with $\lambda, t \in \mathbb{R}$ are defined by

$$
\begin{aligned}
W_{\lambda}^{t} & :=P_{0} V_{\lambda}^{t} P_{0}, \\
R_{\lambda}^{t} & :=P_{1} V_{\lambda}^{t} P_{0} .
\end{aligned}
$$


Moreover, if $\operatorname{dim}\left(\operatorname{ran}\left(P_{0}\right)\right)<\infty$, we define the spectral average $X^{\natural} \in \mathcal{L}(\mathcal{H})$ of an operator $X \in \mathcal{L}(\mathcal{H})$ by

$$
X^{\natural}:=\lim _{T \rightarrow \infty} \frac{1}{2 T} \int_{-T}^{T} \mathrm{~d} t U^{t} P_{0} X P_{0} U^{-t} .
$$

We then have the following result.

Theorem 42 (Van Hove weak coupling limit) Let us assume the validity of the conditions

(a) $\operatorname{dim}\left(\operatorname{ran}\left(P_{0}\right)\right)<\infty$,

(b) $P_{0} A P_{0}=0$ and $P_{1} A P_{1}=0$,

(c) $\int_{0}^{\infty} \mathrm{d} t\left\|P_{0} A P_{1} U^{t} P_{1} A P_{0}\right\|<\infty$.

Then, the following assertions hold.

(1) For $t_{0}>0$ and all $\psi \in \mathcal{H}$, we have

$$
\lim _{\lambda \rightarrow 0} \sup _{t \in\left[0, t_{0}\right]}\left\|U^{-t / \lambda^{2}} W_{\lambda}^{t / \lambda^{2}} P_{0} \psi-\mathrm{e}^{t K^{\natural}} P_{0} \psi\right\|=0,
$$

where $K^{\natural} \in \mathcal{L}(\mathcal{H})$ is the spectral average of $K \in \mathcal{L}(\mathcal{H})$ given by

$$
K:=\int_{0}^{\infty} \mathrm{d} t U^{-t} P_{0} A P_{1} U^{t} P_{1} A P_{0} .
$$

(2) Let $B \in \mathcal{L}(\mathcal{H})$ satisfy $B \geq 0$ and $\left[B, U^{t}\right]=0$ for all $t \in \mathbb{R}$, and let the operator $S_{B} \in \mathcal{L}(\mathcal{H})$ be defined by

$$
S_{B}:=-2 \int_{0}^{\infty} \mathrm{d} t \operatorname{Re}\left[P_{0} A P_{1} B U^{t} P_{1} A P_{0} U^{-t}\right]
$$

where the integral is assumed to converge in norm. For $t_{0}>0$ and all $\varphi, \psi \in \mathcal{H}$, we have

$$
\lim _{\lambda \rightarrow 0} \sup _{t \in\left[0, t_{0}\right]}\left|\left(R_{\lambda}^{t / \lambda^{2}} P_{0} \varphi, B R_{\lambda}^{t / \lambda^{2}} P_{0} \psi\right)-\int_{0}^{t} \mathrm{~d} s\left(\mathrm{e}^{s K^{\natural}} P_{0} \varphi, S_{B}^{\natural} \mathrm{e}^{s K^{\natural}} P_{0} \psi\right)\right|=0 .
$$

Proof. See Davies [13, 14].

Recall from (153) that, for $a \in\{L, R\}$ and $\beta \in \mathbb{R}$, the function $\psi_{a}^{\beta}: \mathbb{R} \rightarrow \mathbb{C}$ is given by

$$
\psi_{a}^{\beta}(t)=\left(\delta_{\mathcal{R}, a}, i_{a} \varrho_{\beta}\left(h_{a}\right) \mathrm{e}^{\mathrm{i} t h_{a}} i_{a}^{*} \delta_{\mathcal{R}, a}\right) .
$$

We then have the following lemma which is used in the proof of Theorem 21. As usual, in the proofs below, the constant $C$ can take different values at each place it appears. Moreover, the Laplace transform at the points $1 \epsilon$ with $\epsilon \in \mathbb{R}$ is denoted by $\Psi_{a}^{\beta}(1 \epsilon):=$ $\int_{0}^{\infty} \mathrm{d} t \psi_{a}^{\beta}(t) \mathrm{e}^{-1 \epsilon t}$ and the temporal Fourier transform by $\hat{\psi}_{a}^{\beta}(\epsilon):=\int_{-\infty}^{\infty} \mathrm{d} t \psi_{a}^{\beta}(t) \mathrm{e}^{-1 \epsilon t}$. 
Lemma 43 (Reservoir time correlation) For $a \in\{L, R\}$ and $\beta \in \mathbb{R}$, there exists a constant $C>0$ s.t., for all $t \in \mathbb{R}$, we have

$$
\left|\psi_{a}^{\beta}(t)\right| \leq C(1+|t|)^{-3 / 2}
$$

Moreover, for all $\epsilon \in \mathbb{R}$, it holds that

$$
\operatorname{Re}\left[\Psi_{a}^{\beta}(1 \epsilon)\right]=\frac{1}{2} \hat{\psi}_{a}^{\beta}(\epsilon)= \begin{cases}2\left(1-\epsilon^{2}\right)^{1 / 2} \varrho_{\beta}(\epsilon), & |\epsilon|<1 \\ 0, & |\epsilon| \geq 1 .\end{cases}
$$

Proof. In order to prove the first assertion, we switch to the energy space representation of the subreservoir Hamiltonians $h_{a}$ from Lemma29already used in the derivation of (162) in order to make the decomposition $\psi_{a}^{\beta}=\frac{2}{\pi}\left(\varphi_{\beta}+\bar{\varphi}_{-\beta}\right)$, where the function $\varphi_{\beta}: \mathbb{R} \rightarrow \mathbb{C}$, independent of $a \in\{L, R\}$, is defined by

$$
\varphi_{\beta}(t):=\int_{0}^{1} \mathrm{~d} e\left(1-e^{2}\right)^{1 / 2} \varrho_{\beta}(e) \mathrm{e}^{1 t e} .
$$

After the coordinate transformation $e \mapsto 1-e$ and one partial integration, we get

$$
\varphi_{\beta}(t)=\frac{1}{2 t}+\frac{\mathrm{e}^{\mathrm{1} t}}{1 t} \sum_{i=1}^{3} \varphi_{\beta, i}(t)
$$

where the functions $\varphi_{\beta, i}: \mathbb{R} \rightarrow \mathbb{C}$ have the form $\varphi_{\beta, i}(t):=\int_{0}^{1} \mathrm{~d} e \phi_{\beta, i}(e) \mathrm{e}^{-1 t e}$, the integrands $\phi_{\beta, i}:(0,1) \rightarrow[0, \infty)$ are defined by

$$
\begin{aligned}
\phi_{\beta, 1}(e) & :=\frac{1}{2} e^{-1 / 2}(2-e)^{1 / 2} \varrho_{\beta}(1-e), \\
\phi_{\beta, 2}(e) & :=\frac{1}{2} e^{1 / 2}(2-e)^{-1 / 2} \varrho_{\beta}(1-e), \\
\phi_{\beta, 3}(e) & :=\beta e^{1 / 2}(2-e)^{1 / 2} \varrho_{\beta}(1-e) \varrho_{-\beta}(1-e),
\end{aligned}
$$

and we used the fact that $\varrho_{\beta}^{\prime}(e)=-\beta \varrho_{\beta}(e) \varrho_{-\beta}(e)$ (the prime denotes the derivative w.r.t. $e$ ). First, using $\left|\phi_{\beta, 1}(e)\right| \leq C e^{-1 / 2}$ for all $e \in(0,1)$, we immediately get $\left|\varphi_{\beta, 1}(t)\right| \leq C$ for all $0 \leq|t| \leq 1$. On the other hand, if $|t|>1$, we write

$$
\varphi_{\beta, 1}(t)=\int_{0}^{1 /|t|} \mathrm{d} e \phi_{\beta, 1}(e) \mathrm{e}^{-1 t e}+\int_{1 /|t|}^{1} \mathrm{~d} e \phi_{\beta, 1}(e) \mathrm{e}^{-1 t e} .
$$

Using directly $\left|\phi_{\beta, 1}(e)\right| \leq C e^{-1 / 2}$ for all $e \in(0,1)$ for the first integral and one partial integration and $\left|\phi_{\beta, 1}^{\prime}(e)\right| \leq C e^{-3 / 2}$ for all $e \in(0,1)$ for the second integral, we can bound the modulus of both integrals by $C|t|^{-1 / 2}$ for $|t|>1$. Moreover, for $i=2,3$, since $\left|\phi_{\beta, i}(e)\right| \leq C$ for all $e \in(0,1)$, we have $\left|\varphi_{\beta, i}(t)\right| \leq C$ for all $0 \leq|t| \leq 1$. If $|t|>1$, using $\left|\phi_{\beta, i}^{\prime}(e)\right| \leq C \mathrm{e}^{-1 / 2}$ for all $e \in(0,1)$ and one partial integration, we get $\left|\varphi_{\beta, i}(t)\right| \leq C|t|^{-1}$. These facts imply 
(307). We next turn to the proof of the second assertion. The real part of the Laplace transform (which exists due to (307)) can be written as

$$
\begin{aligned}
\operatorname{Re}\left[\Psi_{a}^{\beta}(\mathrm{l} \epsilon)\right] & =\frac{1}{2} \int_{-\infty}^{\infty} \mathrm{d} t \psi_{a}^{\beta}(t) \mathrm{e}^{-1 t \epsilon} \\
& =\lim _{\delta \rightarrow 0^{+}} \frac{1}{\pi} \int_{-\infty}^{\infty} \mathrm{d} t \mathrm{e}^{-\delta|t|} \mathrm{e}^{-1 t \epsilon} \int_{-1}^{1} \mathrm{~d} e \xi_{\beta}(e) \mathrm{e}^{1 t e},
\end{aligned}
$$

where, in the first equality, we used that $\psi_{a}^{\beta}(-t)=\bar{\psi}_{a}^{\beta}(t)$ for all $t \in \mathbb{R}$ (leading to a Fourier transform), and, in the second, we set $\xi_{\beta}(e):=\left(1-e^{2}\right)^{1 / 2} \varrho_{\beta}(e)$ and we used (162) and Lebesgue's theorem of dominated convergence. Since $\int_{-\infty}^{\infty} \mathrm{d} t \mathrm{e}^{-\delta|t|} \mathrm{e}^{1 t(e-\epsilon)}=2 \delta /\left(\delta^{2}+(e-\right.$ $\left.\epsilon)^{2}\right)$, using Fubini's theorem, and transforming the coordinates as $e \mapsto(e-\epsilon) / \delta$, we get

$$
\operatorname{Re}\left[\Psi_{a}^{\beta}(1 \epsilon)\right]=\lim _{\delta \rightarrow 0^{+}} \frac{2}{\pi} \int_{-(1+\epsilon) / \delta}^{(1-\epsilon) / \delta} \mathrm{d} e \frac{\xi_{\beta}(\epsilon+\delta e)}{1+e^{2}} .
$$

If $|\epsilon|>1$, using $0 \leq \xi_{\beta}(e) \leq 1$ for all $e \in[-1,1]$, we directly get $\operatorname{Re}\left[\Psi_{a}^{\beta}(1 \epsilon)\right]=0$. For $|\epsilon| \leq 1$, we decompose the numerator as $\xi_{\beta}(\epsilon+\delta e)=\xi_{\beta}(\epsilon)+\left[\xi_{\beta}(\epsilon+\delta e)-\xi_{\beta}(\epsilon)\right]$. Due to $\left|\xi_{\beta}(\epsilon+\delta e)-\xi_{\beta}(\epsilon)\right| \leq C \delta|e|$ for all $e$ in the integration interval, the difference term vanishes and we get $\operatorname{Re}\left[\Psi_{a}^{\beta}(1 \epsilon)\right]=2 \xi_{\beta}(\epsilon)$. Since $\xi_{\beta}( \pm 1)=0$, we arrive at (308).

\section{References}

[1] Alicki R and Fannes M 2001 Quantum dynamical systems (Oxford University Press)

[2] Araki H 1968 On the diagonalization of a bilinear Hamiltonian by a Bogoliubov transformation Publ. RIMS Kyoto Univ. 6 385-442

[3] Araki H 1971 On quasifree states of CAR and Bogoliubov automorphisms Publ. RIMS Kyoto Univ. 6 385-442

[4] Araki $\mathrm{H} 1984$ On the XY-model on two-sided infinite chain Publ. RIMS Kyoto Univ. 20 277-96

[5] Araki $\mathrm{H}$ and $\mathrm{Ho} \mathrm{T} \mathrm{G} 2000$ Asymptotic time evolution of a partitioned infinite two-sided isotropic XY-chain Proc. Steklov Inst. Math. 228 191-204

[6] Aschbacher W H and Pillet C A 2003 Non-equilibrium steady states of the XY chain J. Stat. Phys. $1121153-75$

[7] Aschbacher W H, Jakšić V, Pautrat Y, and Pillet C A 2006 Topics in non-equilibrium quantum statistical mechanics Lecture Notes in Mathematics 1882 (Springer, New York) 1-66 
[8] Aschbacher W H, Jakšić V, Pautrat $\mathrm{Y}$, and Pillet C A 2007 Transport properties of quasi-free fermions J. Math. Phys. 48 032101-1-28

[9] Aschbacher W H and Spohn H 2006 A remark on the strict positivity of the entropy production Lett. Math. Phys. 75 17-23

[10] Böttcher A and Grudsky M S 2005 Spectral properties of banded Toeplitz matrices SIAM (Philadelphia)

[11] Carey A L and Hume L R 1992 Multiple phases and return to equilibrium J. Stat. Phys. 68 787-828

[12] Culvahouse J W, Schinke D P, and Pfortmiller L G 1969 Spin-spin interaction constants from the hyperfine structure of coupled ions Phys. Rev. 177 454-64

[13] Davies E B 1974 Markovian master equations Comm. Math. Phys. 39 91-110

[14] Davies E B 1976 Markovian master equations, // Math. Ann. 219 147-58

[15] D'lorio M, Armstrong R L, and Taylor D R 1983 Longitudinal and transverse spin dynamics of a one-dimensional $X Y$ system studied by chlorine nuclear relaxation in $\mathrm{PrCl}_{3}$ Phys. Rev. B 27 1664-73

[16] Hume L and Robinson D W 1986 Return to equilibrium in the XY model J. Stat. Phys. $44829-48$

[17] Jakšić V, Kritchevski E, and Pillet C A 2006 Mathematical theory of the WignerWeisskopf atom Lecture Notes in Physics 695 (Springer) 145-215

[18] Jakšić V, Ogata Y, and Pillet C A 2006 The Green-Kubo formula for the spin-fermion system Comm. Math. Phys. 268 369-401

[19] Lieb E, Schultz T, and Mattis D 1961 Two soluble models of an antiferromagnetic chain Ann. Physics 16 407-66

[20] Ruelle D 2000 Natural nonequilibrium states in quantum statistical mechanics J. Stat. Phys. 98 57-75

[21] Sologubenko A V, Giannò K, Ott H R, Vietkine A, and Revcolevschi A 2001 Heat transport by lattice and spin excitations in the spin-chain compounds $\mathrm{SrCuO}_{2}$ and $\mathrm{Sr}_{2} \mathrm{CuO}_{3}$ Phys. Rev. B 64 054412-1-11

[22] Spohn H and Lebowitz J 1978 Irreversible thermodynamics for quantum systems weakly coupled to thermal reservoirs Adv. Chem. Phys. 38 109-142

[23] Yafaev D R 1992 Mathematical scattering theory: general theory (AMS) 